\title{
TOWARDS AN EXPLICIT LOCAL JACQUET-LANGLANDS CORRESPONDENCE BEYOND THE CUSPIDAL CASE
}

\author{
by
}

\author{
Vincent Sécherre \& Shaun Stevens
}

\begin{abstract}
We show how the modular representation theory of inner forms of general linear groups over a non-Archimedean local field can be brought to bear on the complex theory in a remarkable way. Let $\mathrm{F}$ be a non-Archimedean locally compact field of residue characteristic $p$, and let $\mathrm{G}$ be an inner form of the general linear group $\mathrm{GL}_{n}(\mathrm{~F}), n \geqslant 1$. We consider the problem of describing explicitly the local Jacquet-Langlands correspondence $\pi \mapsto \mathrm{JL} \pi$ between the complex discrete series representations of $\mathrm{G}$ and $\mathrm{GL}_{n}(\mathrm{~F})$, in terms of type theory. We show that the congruence properties of the local Jacquet-Langlands correspondence exhibited by A. Mínguez and the first named author give information about the explicit description of this correspondence. We prove that the problem of the invariance of the endo-class by the Jacquet-Langlands correspondence can be reduced to the case where the representations $\pi$ and ${ }_{\mathrm{JL}} \pi$ are both cuspidal with torsion number 1 . We also give an explicit description of the Jacquet-Langlands correspondence for all essentially tame discrete series representations of G, up to an unramified twist, in terms of admissible pairs, generalizing previous results by Bushnell and Henniart. In positive depth, our results are the first beyond the case where $\pi$ and ${ }_{\mathrm{JL}} \pi$ are both cuspidal.
\end{abstract}

2010 Mathematics Subject Classification: 22E50

Keywords and Phrases: Jacquet-Langlands correspondence, $\ell$-blocks, Congruences mod $\ell$, Endo-class, Admissible pair, Rectifier.

\section{Introduction}

\section{1 .}

Let $\mathrm{F}$ be a non-Archimedean locally compact field of residue characteristic $p$, let $\mathrm{H}$ be the general linear group $\mathrm{GL}_{n}(\mathrm{~F}), n \geqslant 1$, and let $\mathrm{G}$ be an inner form of $\mathrm{H}$. This is a group of the form $\mathrm{GL}_{m}(\mathrm{D})$, where $m$ divides $n$ and $\mathrm{D}$ is a central division F-algebra whose reduced degree is denoted $d$, with $n=m d$. Let $\mathcal{D}(\mathrm{G}, \mathbf{C})$ denote the set of all isomorphism classes of essentially square integrable, irreducible complex smooth representations of $\mathrm{G}$. The local Jacquet-Langlands correspondence $[\mathbf{2 6}, \mathbf{3 1}, \mathbf{1 8}, \mathbf{1}]$ is a bijection

$$
\begin{aligned}
\mathcal{D}(\mathrm{G}, \mathbf{C}) & \rightarrow \mathcal{D}(\mathrm{H}, \mathbf{C}) \\
\pi & \mapsto \mathrm{JL} \pi
\end{aligned}
$$


specified by a character relation on elliptic regular conjugacy classes. Bushnell and Henniart have elaborated a vast programme aiming at giving an explicit description of this correspondence $[\mathbf{2 3}$, $\mathbf{7}, \mathbf{1 0}, \mathbf{1 3}]$. The present article is a contribution to this programme.

We first have to explain what we mean by an explicit description of the Jacquet-Langlands correspondence. Essentially square integrable representations of $\mathrm{G}$ can be described in terms of parabolic induction. Given such a representation $\pi$, there are a unique integer $r$ dividing $m$ and a cuspidal irreducible representation $\rho$ of $\mathrm{GL}_{m / r}(\mathrm{D})$, unique up to isomorphism, such that $\pi$ is isomorphic to the unique irreducible quotient of the parabolically induced representation

$$
\rho \times \rho \nu^{s(\rho)} \times \cdots \times \rho \nu^{s(\rho)(r-1)}
$$

where $\nu$ is the unramified character "absolute value of the reduced norm" and $s(\rho)$ is a positive integer dividing $d$, associated to $\rho$ in [41]. The essentially square integrable representation $\pi$ is entirely characterized by the pair $(\rho, r)$; this goes back to Bernstein-Zelevinski [44] when D is equal to F, and Tadić [41] in the general case (see also Badulescu [2] when $\mathrm{F}$ has positive characteristic). In particular, we may write $s(\pi)=s(\rho)$. Similarly, associated with the Jacquet-Langlands transfer $\mathrm{JL}_{\mathrm{L}} \pi$, there are an integer $u$ dividing $n$ and a cuspidal irreducible representation $\sigma$ of $\mathrm{GL}_{n / u}(\mathrm{~F})$. The integers $r, u$ are related by the identity $u=r s(\pi)$. It remains to understand how the cuspidal representations $\rho, \sigma$ are related.

Thanks to the theory of simple types, developed by Bushnell and Kutzko [16] for the general linear group $\mathrm{GL}_{n}(\mathrm{~F})$ and by Broussous $[\mathbf{3}]$ and the authors $[\mathbf{3 2}, \mathbf{3 3}, \mathbf{3 4}, \mathbf{3 5}]$ for its inner forms, the cuspidal representation $\rho$ is compactly induced from a compact mod centre, open subgroup. More precisely, there is an extended maximal simple type, made of a compact mod centre subgroup $\mathbf{J}$ of $\mathrm{GL}_{m / r}(\mathrm{D})$ and an irreducible representation $\boldsymbol{\lambda}$ of $\mathbf{J}$, both constructed in a very specific way, such that the compact induction of $\boldsymbol{\lambda}$ to $\mathrm{GL}_{m / r}(\mathrm{D})$ is irreducible and isomorphic to $\rho$. Such a type is uniquely determined up to conjugacy. Giving an explicit description of the local Jacquet-Langlands correspondence will thus consist of describing the extended maximal simple type associated with the representation $\sigma$ in terms of that of $\rho$.

This programme was first carried out for essentially square integrable representations of depth zero, by Silberger-Zink $[\mathbf{3 9}, \mathbf{4 0}]$ and Bushnell-Henniart [14]. Before explaining the other cases which have already been dealt with, we need to introduce two numerical invariants associated to an essentially square integrable, irreducible representation of G. Such a representation $\pi$ has: a torsion number $t(\pi)$, the number of unramified characters $\chi$ of $\mathrm{G}$ such that the twisted representation $\pi \chi$ is isomorphic to $\pi$; and a parametric degree $\delta(\pi)$, defined in [13] via the theory of simple types, which is a multiple of $t(\pi)$ and divides $n$. Both of these integers are invariant under the Jacquet-Langlands correspondence [13]. It is interesting to note that the invariance of the parametric degree implies that $\delta(\pi) s(\pi)=n / r$. Consequently, the representation $\mathrm{JL} \pi$ is cuspidal if and only if the parametric degree of $\pi$ is equal to $n$.

In [13], Bushnell and Henniart treat the case where the cuspidal representation $\pi$ is essentially tame (that is, $\delta(\pi) / t(\pi)$ is prime to $p$ ) and of parametric degree $n$. In that case, they explicitly describe the Jacquet-Langlands correspondence by parametrizing the conjugacy classes of extended maximal simple types in $\mathrm{G}$ and $\mathrm{H}$ by objects called admissible pairs [24]. (We will see these objects in Section 9.) 
In [10], they also treat the case which is in some sense at the opposite extreme to the essentially tame case, where $n$ is of the form $p^{k}$, with $k \geqslant 1$ and $p \neq 2$, and where $\pi$ is a cuspidal representation of $\mathrm{D}^{\times}$which is maximal totally ramified (that is, $\delta(\pi)=n$ and $t(\pi)=1$ ).

In [25], Imai and Tsushima treat the case where $\pi$ is an epipelagic cuspidal representation of $\mathrm{G}$, that is, of depth $1 / n$. Such representations are maximal totally ramified.

With the exception of $[\mathbf{3 9}, \mathbf{4 0}]$ and $[\mathbf{1 4}]$, these results all concern cases where the representations $\pi$ and $\mathrm{JL}_{\mathrm{L}} \pi$ are both cuspidal, that is, when $\pi$ is of parametric degree $n$. In such cases, since the cuspidal representation $\pi$ can be expressed as the compact induction of an extended maximal simple type $(\mathbf{J}, \boldsymbol{\lambda})$, there is a relatively straightforward formula giving the trace of $\pi$ at an elliptic regular element in terms of the trace of $\boldsymbol{\lambda}$ (see [8, Theorem A.14] and [13, (1.2.2)]). The strategies followed in $[\mathbf{1 3}, \mathbf{1 0}]$ and $[\mathbf{2 5}]$ depend crucially on such a formula. When considering a non-cuspidal essentially square integrable representation, we are in a much less favourable situation. For the group $\mathrm{GL}_{n}(\mathrm{~F})$, Broussous [4] and Broussous-Schneider [5] have obtained formulae expressing the trace of such a representation at an elliptic regular element by bringing in the theory of simple types. However, in this article, we follow a different route.

\subsection{Preservation of endo-classes}

An important first step towards the general case is to look at the behavior of the local JacquetLanglands correspondence with respect to endo-classes. An endo-class (over F) is a type-theoretic invariant associated to any essentially square integrable representation of any inner form of any general linear group over $\mathrm{F}$, whose construction requires a considerable machinery $[\mathbf{8}, \mathbf{6}]$. However, for cuspidal representations of $\mathrm{H}$, it turns out to have a rather simple arithmetical interpretation through the local Langlands correspondence [9]. Indeed, two cuspidal irreducible representations of general linear groups over $\mathrm{F}$ have the same endo-class if and only if the irreducible representations of the absolute Weil group $\mathcal{W}_{\mathrm{F}}$ associated to them by the local Langlands correspondence share an irreducible component when restricted to the wild inertia subgroup $\mathcal{P}_{\mathrm{F}}$. The local Langlands correspondence thus induces a bijection between the set of $\mathcal{W}_{\mathrm{F}}$-conjugacy classes of irreducible representations of $\mathcal{P}_{\mathrm{F}}$ and the set $\mathcal{E}(\mathrm{F})$ of endo-classes over $\mathrm{F}$.

It is expected that the local Jacquet-Langlands correspondence preserves endo-classes. More precisely, there is the following conjecture.

Endo-class Invariance Conjecture. For any essentially square integrable, irreducible complex representation $\pi$ of $\mathrm{G}$, the endo-classes of $\pi$ and $\mathrm{JL} \pi$ are the same.

Our first main result is the following (see Theorem 7.1), which reduces this conjecture to the case of maximal totally ramified cuspidal representations.

Theorem A. Assume that, for all $\mathrm{F}$ and $n$, and all cuspidal irreducible complex representations $\pi$ of $\mathrm{G}$ such that $\delta(\pi)=n$ and $t(\pi)=1$, the cuspidal representations $\pi$ and ${ }_{\mathrm{JL}} \pi$ have the same endo-class. Then the Endo-class Invariance Conjecture is true.

Before explaining our strategy, we must first make a detour through the modular representation theory of $\mathrm{G}$ and explain recent developments concerning the modular properties of the Jacquet-Langlands correspondence. Fix a prime number $\ell$ different from $p$, and consider the smooth 
$\ell$-adic representations of $\mathrm{G}$, that is, with coefficients in the algebraic closure $\overline{\mathbf{Q}}_{\ell}$ of the field of $\ell$-adic numbers. There is then the notion of integral irreducible representation of G: containing a G-stable $\overline{\mathbf{Z}}_{\ell}$-lattice (where $\overline{\mathbf{Z}}_{\ell}$ is the ring of integers of $\overline{\mathbf{Q}}_{\ell}$ ), which can then be reduced modulo $\ell$. More precisely, given such a representation $\pi$ containing a stable $\overline{\mathbf{Z}}_{\ell}$-lattice $\Lambda$, Vignéras $[\mathbf{4 2}, \mathbf{4 3}]$ showed that the representation $\Lambda \otimes_{\mathbf{Z}_{\ell}} \overline{\mathbf{F}}_{\ell}$ is smooth of finite length (where $\overline{\mathbf{F}}_{\ell}$ is the residue field of $\overline{\mathbf{Z}}_{\ell}$ ), and its semisimplification is independent of the choice of $\Lambda$; we call this semisimplification the reduction $\bmod \ell$ of $\pi$. Thus we can say that two integral irreducible $\ell$-adic representations of $\mathrm{G}$ are congruent mod $\ell$ if their reductions $\bmod \ell$ are isomorphic.

To relate this to the local Jacquet-Langlands correspondence, we fix an isomorphism of fields between $\mathbf{C}$ and $\overline{\mathbf{Q}}_{\ell}$; replacing one by the other via this isomorphism, we get an $\ell$-adic JacquetLanglands correspondence

$$
\mathcal{D}\left(\mathrm{G}, \overline{\mathbf{Q}}_{\ell}\right) \stackrel{\simeq}{\longrightarrow} \mathcal{D}\left(\mathrm{H}, \overline{\mathbf{Q}}_{\ell}\right)
$$

which is independent of the choice of isomorphism. Thus one can study the compatibility of this correspondence with the relation of congruence mod $\ell$, which was done by Dat $[\mathbf{1 7}]$ and then in full generality by Mínguez and the first author [30]: two integral representations of $\mathcal{D}\left(\mathrm{G}, \overline{\mathbf{Q}}_{\ell}\right)$ are congruent $\bmod \ell$ if and only their images under the $\ell$-adic Jacquet-Langlands correspondence are congruent mod $\ell$ ([30, Théorème 1.1]).

We now need to explain how modular representation theory can give us information on the complex representation theory. The starting point for our strategy to prove Theorem A using modular methods is the fact that two representations of $\mathcal{D}\left(\mathrm{G}, \overline{\mathbf{Q}}_{\ell}\right)$ which are congruent $\bmod \ell$ have the same endo-class. The converse is, of course, not true but we will see that one can nevertheless link two essentially square integrable representations with the same endo-class by a chain of congruence relations. Let us explain this in more detail.

Firstly, for any irreducible $\ell$-adic representation of $\mathrm{G}$, we have a notion of mod- $\ell$ inertial supercuspidal support (see Definition 4.1, and also [22] in the split case), coming from the notion of supercuspidal support for irreducible representations of $G$ with coefficients in $\overline{\mathbf{F}}_{\ell}$, defined in [27]. Two irreducible complex representations of $\mathrm{G}$ are said to be $\ell$-linked (Definitions 5.1 and 4.2) if there is a field isomorphism $\mathbf{C} \simeq \overline{\mathbf{Q}}_{\ell}$ such that the resulting irreducible $\ell$-adic representations have the same mod- $\ell$ inertial supercuspidal support. This is independent of the choice of field isomorphism and it is not hard, using the work done in [30], to show that the Jacquet-Langlands correspondence preserves the relation of being $\ell$-linked for essentially square-integrable representations (Propositions 6.1 and 6.2). We can now introduce the following definition (Definition 5.6).

Definition. Two irreducible complex representations $\pi, \pi^{\prime}$ of $\mathrm{G}$ are said to be linked if there are a finite sequence of prime numbers $\ell_{1}, \ldots, \ell_{r}$, all different from $p$, and a finite sequence of irreducible complex representations $\pi=\pi_{0}, \pi_{1}, \ldots, \pi_{r}=\pi^{\prime}$ such that, for each $i \in\{1, \ldots, r\}$, the representations $\pi_{i-1}$ and $\pi_{i}$ are $\ell_{i}$-linked.

Two essentially square integrable complex representations which are linked have the same endo-class. More generally, if we define the semi-simple endo-class of an irreducible representation to be the weighted formal sum of the endo-classes of the cuspidal representations in its cuspidal support (with multiplicities determined by the sizes of the groups - see (5.2)), then two irreducible representations which are linked have the same semi-simple endo-class. The interest 
of the definition is apparent from the following theorem (see Theorem 5.10), which says that the converse is also true.

Theorem B. Two irreducible complex representations of $\mathrm{G}$ are linked if and only if they have the same semi-simple endo-class.

In particular, two essentially square integrable complex representations have the same endoclass if and only if they are linked; moreover, one can then link them by a sequence of essentially square integrable representations (Remark 5.9).

Theorem B gives a remarkable reinterpretation of what it means for two irreducible complex representations to have the same semi-simple endo-class. Beyond the intrinsic interest in explicating the notion of endo-class and its relation with modular representation theory, the main interest in this reformulation comes from the fact that, applying results from [30], we are able to prove the following (Theorem 6.3).

Theorem C. Two essentially square integrable complex representations of $\mathrm{G}$ are linked if and only if their transfers to $\mathrm{H}$ are linked.

It follows from Theorems B and $\mathrm{C}$ that two essentially square integrable complex representations of $\mathrm{G}$ have the same endo-class if and only if their transfers to $\mathrm{H}$ have the same endo-class. Thus, denoting by $\mathcal{E}_{n}(\mathrm{~F})$ the set of endo-classes over $\mathrm{F}$ of degree dividing $n$, the Jacquet-Langlands correspondence induces a bijection

$$
\pi_{1}: \mathcal{E}_{n}(\mathrm{~F}) \rightarrow \mathcal{E}_{n}(\mathrm{~F})
$$

We now observe the following fact (Proposition 6.5).

Proposition. For every essentially square integrable complex representation of $\mathrm{G}$, there is a cuspidal complex representation of $\mathrm{G}$ with the same endo-class and with parametric degree $n$.

To prove the conjecture - that is, to prove that $\boldsymbol{\pi}_{1}$ is the identity map - it is therefore sufficient to prove that, for every cuspidal complex representation $\pi$ of $\mathrm{G}$ of parametric degree $n$, the representations $\pi$ and ${ }_{\mathrm{JL}} \pi$ have the same endo-class. Using techniques developed in [13, Section 6], we can go further and show that one need only consider cuspidal representations of parametric degree $n$ and torsion number 1, thus obtaining Theorem A. Therefore, to prove the Endo-class Invariance Conjecture, it remains only to prove the following conjecture. Say that an endo-class is totally ramified if it has residual degree 1, that is, if its tame parameter field (in the sense of $[\mathbf{1 5}$, Section 2]) is totally ramified.

Conjecture. For all $\mathrm{F}$ and $n$, and for every totally ramified $\mathrm{F}$-endo-class $\Theta$ of degree n, there is a cuspidal complex representation $\pi$ of $\mathrm{G}$ with endo-class $\boldsymbol{\Theta}$ such that ${ }_{\mathrm{JL}} \pi$ has endo-class $\boldsymbol{\Theta}$.

This conjecture is known to be true in all the cases where the explicit correspondence is known (see $§ 1.1$. See also the remark at the end of this introduction for more recent developments. 


\subsection{The level zero part}

We now leave to one side the preservation of endo-classes and pass to the next step towards an explicit description of the Jacquet-Langlands correspondence. We will see that the modular methods described in the previous paragraphs can be pushed further to yield additional information. Let $\Theta$ be an endo-class of degree dividing $n$ and suppose that it is invariant under the Jacquet-Langlands correspondence, i.e. $\boldsymbol{\pi}_{1}(\boldsymbol{\Theta})=\boldsymbol{\Theta}$. (See the remark at the end of this introduction for a discussion about this assumption.) The correspondence thus induces a bijection between isomorphism classes of essentially square integrable complex representations of $\mathrm{G}$ with endo-class $\boldsymbol{\Theta}$, and those of $\mathrm{H}$. Since the correspondence is also compatible with unramified twisting, we get a bijection

$$
\mathcal{D}_{0}(\mathrm{G}, \boldsymbol{\Theta}) \stackrel{\simeq}{\longrightarrow} \mathcal{D}_{0}(\mathrm{H}, \boldsymbol{\Theta})
$$

where $\mathcal{D}_{0}(\mathrm{G}, \boldsymbol{\Theta})$ denotes the set of inertial classes of essentially square integrable complex representations of $G$ with endo-class $\Theta$. The theory of simple types $[\mathbf{1 6}, \mathbf{3 4}, \mathbf{3 5}, \mathbf{3 6}]$ gives us a canonical bijection between $\mathcal{D}_{0}(G, \Theta)$ and the set $\mathcal{T}(G, \Theta)$ of G-conjugacy classes of simple types for $\mathrm{G}$ with endo-class $\Theta$. More precisely, the inertial class of an essentially square integrable complex representation $\pi$ corresponds to the conjugacy class of a simple type $(\mathrm{J}, \lambda)$, formed of a compact open subgroup $\mathrm{J}$ of $\mathrm{G}$ and an irreducible representation $\lambda$ of $\mathrm{J}$, if and only if $\lambda$ is an irreducible component of the restriction of $\pi$ to J. Thus we get a bijection

$$
\mathcal{T}(\mathrm{G}, \boldsymbol{\Theta}) \stackrel{\simeq}{\longrightarrow} \mathcal{T}(\mathrm{H}, \boldsymbol{\Theta}) \text {. }
$$

To go further, we need to enter into the detail of the structure of simple types (Paragraph 3.3).

Given a simple type $(\mathrm{J}, \lambda)$ of $\mathrm{G}$ with endo-class $\boldsymbol{\Theta}$, the group $\mathrm{J}$ contains a unique maximal normal pro- $p$ subgroup, denoted $\mathrm{J}^{1}$. The restriction of $\lambda$ to $\mathrm{J}^{1}$ is isotypic, that is, it is a direct sum of copies of a single irreducible representation $\eta$. This representation $\eta$ can be extended to a representation of $\mathrm{J}$ with the same intertwining set as $\eta$. If we fix such an extension $\kappa$, then the representation $\lambda$ can be expressed in the form $\kappa \otimes \sigma$, where $\sigma$ is an irreducible representation of $\mathrm{J}$, trivial on $\mathrm{J}^{1}$.

The quotient group $\mathrm{J} / \mathrm{J}^{1}$ is (non-canonically) isomorphic to a product of copies of a single general linear group over a finite field $\boldsymbol{d}$ and $\sigma$, viewed as a representation of such a product, is the tensor product of copies of a single cuspidal representation. A theorem of Green [21] allows us to parametrize $\sigma$ by a character of $\boldsymbol{k}^{\times}$, where $\boldsymbol{k}$ is a suitable extension of $\boldsymbol{d}$. This character is determined up to conjugation by the Galois group of $\boldsymbol{k}$ over a certain subfield $\boldsymbol{e}$ of $\boldsymbol{d}$.

We denote by $\mathrm{X}$ the group of characters of $\boldsymbol{k}^{\times}$and by $\Gamma$ the Galois group $\operatorname{Gal}(\boldsymbol{k} / \boldsymbol{e})$. Fixing once and for all a choice of representation $\kappa$ for a maximal simple type in $\mathrm{G}$ with endo-class $\boldsymbol{\Theta}$, we get a bijection from $\mathrm{X} / \Gamma$ to $\mathcal{T}(\mathrm{G}, \boldsymbol{\Theta})$ (see Paragraph 3.3 for details). Making a similar choice for $\mathrm{H}$, we also get a bijection from $\mathrm{X} / \Gamma$ to $\mathcal{T}(\mathrm{H}, \boldsymbol{\Theta})$. Composing with (1.1), we get a permutation

$$
\Upsilon: \mathrm{X} / \Gamma \rightarrow \mathrm{X} / \Gamma
$$

which depends on various choices (see Paragraph 8.1). Although one could fix choices, it is not clear which are the natural ones in general so we must take care with them. In particular, we will see that, in the essentially tame case, one can make sense of the notion of a compatible choice for $\mathrm{G}$ and $\mathrm{H}$. 
We write $[\alpha]$ for the $\Gamma$-orbit of a character $\alpha \in \mathrm{X}$. The following result (see Proposition 8.8), which again is proved via modular methods, suggests that, in order to determine the permutation $\Upsilon$ it is sufficient to compute the value of $\Upsilon([\alpha])$ for certain characters $\alpha$ only.

Proposition. Let $\alpha \in \mathrm{X}$ and let $\boldsymbol{l}$ be the unique subfield of $\boldsymbol{k}$ such that the stabilizer of $\alpha$ in $\Gamma$ is $\operatorname{Gal}(\boldsymbol{k} / \boldsymbol{l})$. Suppose there are a $\Gamma$-regular character $\beta \in \mathrm{X}$ and a prime number $\ell \neq p$ prime to the order of $\boldsymbol{l}^{\times}$such that the order of $\beta \alpha^{-1}$ is a power of $\ell$. Suppose further that $\Upsilon([\beta])=[\beta \mu]$, for some character $\mu \in \mathrm{X}$. Then $\Upsilon([\alpha])=[\alpha \nu]$ where $\nu \in \mathrm{X}$ is the unique character of order prime to $\ell$ such that $\mu \nu^{-1}$ has order a power of $\ell$.

In fact we need a more powerful version of this result, which we do not explain here, which requires being able to pass from $\mathrm{G}$ to a bigger group $\mathrm{GL}_{m^{\prime}}(\mathrm{D})$, with $m^{\prime}>m$. (See Section 8, in particular Paragraph 8.3.)

To conclude, in the final section of the paper, we illustrate this principle in the essentially tame case. We start from the Parametrization Theorem [13, 6.1], which gives a canonical bijection

$$
(\mathrm{L} / \mathrm{F}, \xi) \mapsto \Pi(\mathrm{G}, \xi)
$$

between isomorphism classes of admissible pairs of degree $n$ and isomorphism classes of essentially tame cuspidal irreducible representations of $\mathrm{G}$ of parametric degree $n$. The First Comparison Theorem [13, 6.1] shows how to translate the Jacquet-Langlands correspondence for these cuspidal representations in terms of admissible pairs: for any admissible pair $(\mathrm{L} / \mathrm{F}, \xi)$ of degree $n$, there is a canonically determined tamely ramified character $\nu$ of $\mathrm{L}^{\times}$such that $\nu^{2}=1$ and

$$
\mathrm{JL} \Pi(\mathrm{G}, \xi)=\Pi(\mathrm{H}, \xi \nu) .
$$

We show that, for appropriate choices, this result can be rephrased in terms of our $\alpha$-parameters and gives us an explicit formula for $\Upsilon([\alpha])$ for all $\Gamma$-regular characters $\alpha \in \mathrm{X}$. Applying the proposition above, we then prove that this explicit formula actually holds for any $\alpha \in \mathrm{X}$.

As in $[\mathbf{1 3}]$, we formulate our result in terms of admissible pairs. We first define a bijection

$$
[\mathrm{L} / \mathrm{F}, \xi] \mapsto \Pi_{0}[\mathrm{G}, \xi]
$$

between inertial classes of admissible pairs (see Definition 9.5) of degree dividing $n$ and inertial classes of discrete series representations of $\mathrm{G}$ with essentially tame endo-class, extending (1.2) up to inertia. In the case where $\mathrm{G}$ is the group $\mathrm{H}$, this bijection is canonical, but for a general $\mathrm{G}$ it depends a priori on various choices. We prove the following result (see Theorem 9.13).

Theorem D. Let $[\mathrm{L} / \mathrm{F}, \xi]$ be an inertial class of admissible pairs of degree dividing $n$. There is a canonically determined tamely ramified character $\mu$ of the group of units of the ring of integers of $\mathrm{L}$ such that $\mu^{2}=1$ and

$$
\mathrm{JL}_{0}[\mathrm{G}, \xi]=\Pi_{0}[\mathrm{H}, \xi \mu] .
$$

We thus deduce a posteriori that our bijection (1.3) is canonical, that is, it does not depend on the various choices we have made (see Remark 9.15). 
Remark. After this paper was written, A. Dotto proved the Endo-class Invariance Conjecture in [20], using methods developed here and in [13]. He goes further and gives an explicit description of the Jacquet-Langlands correspondence up to inertia.

Acknowledgements. The research of the second author was supported by the Engineering and Physical Sciences Research Council (grant EP/H00534X/1). We also thank the anonymous referee for several useful comments, particularly relating to the presentation in Section 6 .

\section{Notation}

We fix a non-Archimedean locally compact field $\mathrm{F}$ with residual characteristic $p$. Write $q$ for the cardinality of the residue field of $\mathrm{F}$.

Given D a finite dimensional central division F-algebra and a positive integer $m \geqslant 1$, we write $\mathrm{M}_{m}(\mathrm{D})$ for the algebra of $m \times m$ matrices with coefficients in $\mathrm{D}$ and $\mathrm{GL}_{m}(\mathrm{D})$ for the group of its invertible elements. Choose an $m \geqslant 1$ and write $\mathrm{G}=\mathrm{GL}_{m}(\mathrm{D})$. Write $d$ for the reduced degree of $\mathrm{D}$ over $\mathrm{F}$, and define $n=m d$.

Given an algebraically closed field $\mathrm{R}$ of characteristic different from $p$, we will consider smooth representations of the locally profinite group $G$ with coefficients in $R$. We write $\operatorname{Irr}(G, R)$ for the set of isomorphism classes of irreducible representations of $G$ and $\mathcal{R}(G, R)$ for the Grothendieck group of its finite length representations, identified with the free abelian group with basis $\operatorname{Irr}(\mathrm{G}, \mathrm{R})$. If $\pi$ is a representation of $\mathrm{G}$, the integer $m$ is called its degree.

Given $\alpha=\left(m_{1}, \ldots, m_{r}\right)$ a family of positive integers of sum $m$, we write $\boldsymbol{i}_{\alpha}$ for the functor of standard parabolic induction associated with $\alpha$, normalized with respect to the choice of a square root in the field $\mathrm{R}$ of the cardinality $q$ of the residual field of $\mathrm{F}$. Given, for each $i \in\{1, \ldots, r\}$, a representation $\pi_{i}$ of $\mathrm{GL}_{m_{i}}(\mathrm{D})$, we write

$$
\pi_{1} \times \cdots \times \pi_{r}=\boldsymbol{i}_{\alpha}\left(\pi_{1} \otimes \cdots \otimes \pi_{r}\right) .
$$

Given a representation $\pi$ and a character $\chi$ of $\mathrm{G}$, we write $\pi \chi$ for the twisted representation defined by $g \mapsto \chi(g) \pi(g)$.

We fix once and for all a smooth additive character $\psi: \mathrm{F} \rightarrow \mathrm{R}^{\times}$, trivial on the maximal ideal $\mathfrak{p}$ of the ring of integers $\mathcal{O}$ of $\mathrm{F}$ but not trivial on $\mathcal{O}$.

We write $\nu$ for the unramified R-character of $\mathrm{G}$ given by composing the reduced norm from $\mathrm{G}$ to $\mathrm{F}^{\times}$with the absolute value of $\mathrm{F}$ which takes any uniformizer to the inverse of $q$ in $\mathrm{R}$.

\section{Preliminaries}

In this section, we let $\mathrm{R}$ be an algebraically closed field of characteristic different from $p$.

\section{1 .}

Let $\rho$ be a cuspidal irreducible R-representation of G. Associated with $\rho$, there is a positive integer $s(\rho)$ defined in [28, Paragraph 3.4] (see also Remark 3.8). When $\mathrm{R}$ is the field of complex numbers, $s(\rho)$ is the unique positive integer $k$ such that $\rho \times \rho \nu^{k}$ is reducible, and it is related to 
the parametric degree $\delta(\rho)$ defined in $[\mathbf{1 3}$, Section 2] by the formula $s(\rho) \delta(\rho)=n$. For the general case, see Remark 3.8.

In $[\mathbf{2 7}]$ we attach to $\rho$ and any integer $r \geqslant 1$ an irreducible subrepresentation $\mathrm{Z}(\rho, r)$ and an irreducible quotient $\mathrm{L}(\rho, r)$ of the induced representation

$$
\rho \times \rho \nu_{\rho} \times \cdots \times \rho \nu_{\rho}^{r-1}
$$

(see [27, Paragraph 7.2 and Définition 7.5]), where $\nu_{\rho}$ is the character $\nu^{s(\rho)}$.

When $\mathrm{R}$ is the field of complex numbers, $\mathrm{Z}(\rho, r)$ and $\mathrm{L}(\rho, r)$ are uniquely determined in this way, and all essentially square integrable representations of $\mathrm{G}$ are isomorphic to a representation of the form $\mathrm{L}(\rho, r)$ for a unique pair $(\rho, r)$.

For an arbitrary $\mathrm{R}$, the representation $\mathrm{L}(\rho, r)$ is called a discrete series $\mathrm{R}$-representation of $\mathrm{G}$ and $\mathrm{Z}(\rho, r)$ is called a Speh $\mathrm{R}$-representation. If $\rho$ is supercuspidal, $\mathrm{Z}(\rho, r)$ is called a super-Speh representation.

According to [27, Paragraph 8.1], where the notion of residually nondegenerate representation is defined, the induced representation (3.1) contains a unique residually nondegenerate irreducible subquotient, denoted

$$
\operatorname{Sp}(\rho, r) .
$$

When $\mathrm{R}$ has characteristic 0 , this is equal to $\mathrm{L}(\rho, r)$. When $\mathrm{R}$ has characteristic $\ell>0$ however, it may differ from $\mathrm{L}(\rho, r)$ (see [27, Remark 8.14]).

Assume $\mathrm{R}$ has characteristic $\ell>0$, and let us write $\omega(\rho)$ for the smallest positive integer $i \geqslant 1$ such that $\rho \nu_{\rho}^{i}$ is isomorphic to $\rho$. Then the irreducible representation

$$
\operatorname{Sp}\left(\rho, \omega(\rho) \ell^{v}\right)
$$

is cuspidal for any integer $v \geqslant 0$. Moreover, any cuspidal non-supercuspidal irreducible representation is of the form (3.2) for a supercuspidal irreducible representation $\rho$ and a unique integer $v \geqslant 0$ (see [27, Théorème 6.14]). We record this latter fact for future reference.

Proposition 3.1. - Assume $\mathrm{R}$ has positive characteristic $\ell$, and let $\rho$ be a cuspidal irreducible representation of $\mathrm{G}$. There are a unique positive integer $k=k(\rho)$ and a supercuspidal irreducible representation $\tau$ of degree $m / k$ such that $\rho$ is isomorphic to $\operatorname{Sp}(\tau, k)$.

\section{2 .}

In this paragraph, we assume that $\mathrm{R}$ is an algebraic closure $\overline{\mathbf{Q}}_{\ell}$ of the field of $\ell$-adic numbers. Recall (see $[\mathbf{4 2}]$ ) that an irreducible $\ell$-adic representation of $\mathrm{G}$ is integral if it contains a G-stable

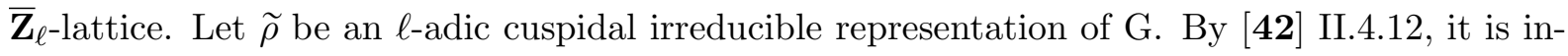
tegral if and only if its central character has values in $\overline{\mathbf{Z}}_{\ell}$. In particular, there is always an unramified twist of $\tilde{\rho}$ which is integral.

Assume $\tilde{\rho}$ is integral and write $a=a(\widetilde{\rho})$ for the length of its reduction $\bmod \ell$, denoted $\mathbf{r}_{\ell}(\widetilde{\rho})$.

Proposition 3.2 ([28, Theorem 3.15]). - Let $\rho$ be an irreducible factor of $\mathbf{r}_{\ell}(\widetilde{\rho})$. Then

$$
\mathbf{r}_{\ell}(\widetilde{\rho})=\rho+\rho \nu+\cdots+\rho \nu^{a-1},
$$

where $\nu$ denotes the unramified mod $\ell$ character "absolute value of the reduced norm". 


\section{3.}

We recall briefly the language of simple strata, though we do not require much of the detail of the constructions. For a detailed presentation, see $[\mathbf{3 4}, \mathbf{2 8}]$. For simple strata, we use the simplified notation of [15, Chapter 2].

Let $[\mathfrak{a}, \beta]$ be a simple stratum in the simple central F-algebra $\mathrm{M}_{m}(\mathrm{D})$. We don't recall the precise definition: we simply recall that it is made of an element $\beta \in \mathrm{M}_{m}(\mathrm{D})$ such that the F-algebra $\mathrm{F}[\beta]$ is a field, and a hereditary order $\mathfrak{a} \subseteq \mathrm{M}_{m}(\mathrm{D})$ normalized by $\mathrm{F}[\beta]^{\times}$. The centralizer of $\beta$ in $\mathrm{M}_{m}(\mathrm{D})$, denoted $\mathrm{B}$, is a simple central $\mathrm{F}[\beta]$-algebra. There are an $\mathrm{F}[\beta]$-division algebra $\mathrm{D}^{\prime}$ and an integer $m^{\prime} \geqslant 1$ such that

$$
\mathrm{B} \simeq \mathrm{M}_{m^{\prime}}\left(\mathrm{D}^{\prime}\right)
$$

The intersection $\mathfrak{b}=\mathfrak{a} \cap \mathrm{B}$ is a hereditary order in B.

Recall $[\mathbf{3 2}, \mathbf{2 8}]$ that, associated with $[\mathfrak{a}, \beta]$, there are compact open subgroups

$$
\mathrm{H}^{1}(\mathfrak{a}, \beta) \subseteq \mathrm{J}^{1}(\mathfrak{a}, \beta) \subseteq \mathrm{J}(\mathfrak{a}, \beta)
$$

of $\mathrm{G}$, together with a non-empty finite set $\mathcal{C}(\mathfrak{a}, \beta)$ depending on the choice of $\psi$ made in Section 2. These groups are normal in $\mathrm{J}(\mathfrak{a}, \beta)$, and the elements of $\mathfrak{C}(\mathfrak{a}, \beta)$ are R-characters of $\mathrm{H}^{1}(\mathfrak{a}, \beta)$, called simple characters. Besides, $\mathrm{H}^{1}(\mathfrak{a}, \beta)$ and $\mathrm{J}^{1}(\mathfrak{a}, \beta)$ are pro- $p$-groups, and $\mathrm{J}(\mathfrak{a}, \beta)$ is equal to $\mathfrak{b}^{\times} \mathrm{J}^{1}(\mathfrak{a}, \beta)$.

Attached to a simple character $\theta \in \mathfrak{C}(\mathfrak{a}, \beta)$ there is an invariant called its endo-class. We will not recall the precise definition of this invariant, which can be found in $[\mathbf{8}, \mathbf{6}]$. We will only need a few properties of endo-classes, which we will recall when they are needed. Endo-classes form a set $\mathcal{E}(\mathrm{F})$ which depends only on $\mathrm{F}$.

Lemma 3.3 ([6, Lemma 4.7]). - Given a simple character $\theta \in \mathcal{C}(\mathfrak{a}, \beta)$ with endo-class $\boldsymbol{\Theta}$, the degree, ramification index and residue degree of $\mathrm{F}[\beta]$ over $\mathrm{F}$ only depend on $\boldsymbol{\Theta}$. These integers are called the degree, ramification index and residue degree of $\boldsymbol{\Theta}$, respectively.

The endo-class of a simple character in $\mathrm{G}$ has degree dividing $n$. Conversely, any endo-class of degree dividing $n$ occurs as the endo-class of some simple character in G.

A $\beta$-extension of a simple character $\theta \in \mathfrak{C}(\mathfrak{a}, \beta)$ is an irreducible representation of $\mathrm{J}(\mathfrak{a}, \beta)$ with coefficients in $\mathrm{R}$ whose restriction to $\mathrm{J}^{1}(\mathfrak{a}, \beta)$ is irreducible, whose restriction to $\mathrm{H}^{1}(\mathfrak{a}, \beta)$ contains $\theta$ and which is intertwined by any element of $\mathrm{B}^{\times}$(see $[\mathbf{3 3}, \mathbf{2 8}]$ ).

Assume now that $\mathfrak{b}$ is a maximal order in $\mathrm{B}$, in which case we say the simple stratum $[\mathfrak{a}, \beta]$, the simple characters in $\mathcal{C}(\mathfrak{a}, \beta)$ and their $\beta$-extensions are maximal. Let us fix an isomorphism (3.3) such that the image of $\mathfrak{b}$ is the maximal order made of all matrices with integer entries. There is a natural group isomorphism

$$
\mathrm{J}(\mathfrak{a}, \beta) / \mathrm{J}^{1}(\mathfrak{a}, \beta) \simeq \mathrm{GL}_{m^{\prime}}(\boldsymbol{d})
$$

where $\boldsymbol{d}$ is the residue field of $\mathrm{D}^{\prime}$. We write $\mathcal{G}$ for the group on the right hand side. Let us fix a $\beta$-extension $\kappa$ of some simple character $\theta \in \mathcal{C}(\mathfrak{a}, \beta)$. We write $\mathrm{J}=\mathrm{J}(\mathfrak{a}, \beta)$ and $\mathrm{J}^{1}=\mathrm{J}^{1}(\mathfrak{a}, \beta)$.

We fix a finite extension $\boldsymbol{k}$ of $\boldsymbol{d}$ of degree $m^{\prime}$. We write $\Sigma$ for the Galois group of this extension and X for the group of R-characters of $\boldsymbol{k}^{\times}$. Given $\alpha \in \mathrm{X}$, there is a unique subfield $\boldsymbol{d} \subseteq \boldsymbol{d}[\alpha] \subseteq \boldsymbol{k}$ 
such that the $\Sigma$-stabilizer of $\alpha$ is $\operatorname{Gal}(\boldsymbol{k} / \boldsymbol{d}[\alpha])$, and then a character $\alpha_{0}$ of $\boldsymbol{d}[\alpha]^{\times}$such that $\alpha$ is equal to $\alpha_{0}$ composed with the norm of $\boldsymbol{k}$ over $\boldsymbol{d}[\alpha]$. If we write $u$ for the degree of $\boldsymbol{d}[\alpha]$ over $\boldsymbol{d}$, then $\alpha_{0}$ defines a supercuspidal irreducible R-representation $\sigma_{0}$ of $\mathrm{GL}_{u}(\boldsymbol{d})$ - see [21] if $\mathrm{R}$ has characteristic 0 , and $[\mathbf{1 9}]$ or $[\mathbf{2 9}]$ otherwise.

Remark 3.4. - More precisely, if R has characteristic 0, fix an embedding of $\boldsymbol{d}[\alpha]$ in $\mathrm{M}_{u}(\boldsymbol{d})$. Then $\sigma_{0}$ is the unique (up to isomorphism) irreducible representation of $\mathrm{GL}_{u}(\boldsymbol{d})$ such that

$$
\operatorname{tr} \sigma_{0}(g)=(-1)^{u-1} \cdot \sum_{\gamma} \alpha_{0}^{\gamma}(g),
$$

for all $g \in \boldsymbol{d}[\alpha]^{\times}$of degree $u$ over $\boldsymbol{d}$, where $\gamma$ runs over $\operatorname{Gal}(\boldsymbol{d}[\alpha] / \boldsymbol{d})$.

The character $\alpha \in \mathrm{X}$ thus defines a supercuspidal R-representation

$$
\boldsymbol{\sigma}(\alpha)=\sigma_{0} \otimes \cdots \otimes \sigma_{0}
$$

of the Levi subgroup $\mathrm{GL}_{u}(\boldsymbol{d}) \times \cdots \times \mathrm{GL}_{u}(\boldsymbol{d})$ in $\mathcal{G}$. Moreover, the fibers of the map $\alpha \mapsto \boldsymbol{\sigma}(\alpha)$ are the $\Sigma$-orbits of X. Write $r$ for the integer defined by $r u=m^{\prime}$. The maximal order $\mathfrak{b}$ contains a unique principal order $\mathfrak{b}_{r}$ of period $r$ whose image under (3.3) consists of matrices with entries in the ring of integers of $\mathrm{D}^{\prime}$ whose reduction modulo its maximal ideal is upper triangular by blocks of size $r$. We write $\mathfrak{a}_{r}$ for the unique order normalized by $\mathrm{F}[\beta]^{\times}$such that $\mathfrak{a}_{r} \cap \mathrm{B}=\mathfrak{b}_{r}$, and $\kappa_{r}$ for the transfer of $\kappa$ with respect to the simple stratum $\left[\mathfrak{a}_{r}, \beta\right]$ in the sense of [28, Proposition 2.3]. Considering $\boldsymbol{\sigma}(\alpha)$ as a representation of the group $\mathrm{J}_{r}=\mathrm{J}\left(\mathfrak{a}_{r}, \beta\right)$ trivial on $\mathrm{J}^{1}\left(\mathfrak{a}_{r}, \beta\right)$, we define

$$
\boldsymbol{\lambda}(\alpha)=\kappa_{r} \otimes \boldsymbol{\sigma}(\alpha)
$$

which is a simple supertype in $\mathrm{G}$ defined on $\mathrm{J}_{r}$ in the sense of [37]. Write $\Gamma$ for the Galois group of $\boldsymbol{k}$ over $\boldsymbol{e}$, where $\boldsymbol{e}$ denotes the residue field of $\mathrm{F}[\beta]$.

Write $\Theta$ for the endo-class of the simple character $\theta \in \mathcal{C}(\mathfrak{a}, \beta)$, and $\mathcal{T}(\mathrm{G}, \boldsymbol{\Theta}, \mathrm{R})$ for the set of isomorphism classes of simple $\mathrm{R}$-supertypes in $\mathrm{G}$ with endo-class $\boldsymbol{\Theta}$, that is, simple R-supertypes whose associated simple character has endo-class $\Theta$.

Recall ([37] Definition 6.1) that two simple R-supertypes in $\mathrm{G}$ are said to be equivalent if the representations of $\mathrm{G}$ obtained from them by compact induction are isomorphic.

Proposition 3.5. - The map

$$
\alpha \mapsto \lambda(\alpha)
$$

induces a surjection from $\mathrm{X}$ onto the set of equivalence classes of $\mathcal{T}(\mathrm{G}, \boldsymbol{\Theta}, \mathrm{R})$. The fibers of this map are the $\Gamma$-orbits of $\mathrm{X}$.

Proof. - Surjectivity follows from the definition of a simple supertype [37, Paragraph 2.2] and the fact that any supercuspidal irreducible R-representation of $\mathcal{G}$ is of the form $\boldsymbol{\sigma}(\alpha)$ for some $\alpha \in \mathrm{X}$ with trivial $\Sigma$-stabilizer.

The description of the fibers follows from [36, Theorem 7.2] together with the fact that the map $\alpha \mapsto \boldsymbol{\sigma}(\alpha)$ is $\Gamma$-equivariant, with fibers the $\Sigma$-orbits of X. Note that [36] is written for complex representations, but [36, Theorem 7.2] holds true in any characteristic different from $p$. 
Proposition 3.6. - The bijection

$$
\{\Gamma \text {-orbits of } \mathrm{X}\} \leftrightarrow\{\text { equivalence classes of } \mathcal{T}(\mathrm{G}, \boldsymbol{\Theta}, \mathrm{R})\}
$$

depends only on the choice of $\kappa$, not on that of the isomorphism (3.3).

Proof. - Choosing another isomorphism $\mathrm{B} \simeq \mathrm{M}_{m^{\prime}}\left(\mathrm{D}^{\prime}\right)$ such that the image of $\mathfrak{b}$ is the maximal order made of all matrices with integer entries has the effect - according to the Skolem-Noether theorem - of conjugating by an element $g \in \mathrm{GL}_{m^{\prime}}\left(\mathrm{D}^{\prime}\right)$ normalizing this standard maximal order. Thus, if $\boldsymbol{\sigma}^{\prime}(\alpha)$ is the representation of $\mathrm{J}_{r}$ trivial on $\mathrm{J}^{1}\left(\mathfrak{a}_{r}, \beta\right)$ corresponding to $\alpha$ with respect to that choice of isomorphism, it differs from $\boldsymbol{\sigma}(\alpha)$ by conjugating by $g$.

Remark 3.7. - Suppose $\boldsymbol{k}_{1}$ is another extension of $\boldsymbol{d}$ of degree $m^{\prime}$. Write $\mathrm{X}_{1}$ for the group of $\mathrm{R}$-characters of its invertible elements and $\Gamma_{1}$ for the Galois group $\operatorname{Gal}\left(\boldsymbol{k}_{1} / \boldsymbol{e}\right)$. Let $\boldsymbol{t}$ denote the bijection (3.5) and write $\boldsymbol{t}_{1}$ for its analogue obtained by replacing $\boldsymbol{k}$ by $\boldsymbol{k}_{1}$. Choosing an isomorphism of $\boldsymbol{e}$-algebras $\boldsymbol{k} \rightarrow \boldsymbol{k}_{1}$ induces a bijection

$$
\boldsymbol{b}: \mathrm{X}_{1} / \Gamma_{1} \rightarrow \mathrm{X} / \Gamma
$$

which does not depend on this choice, and one has $\boldsymbol{t}_{1}=\boldsymbol{t} \circ \boldsymbol{b}$.

\section{4.}

Recall [28] that any supercuspidal R-representation $\rho$ of $\mathrm{G}$ contains a maximal simple character, uniquely determined up to G-conjugacy. We define the endo-class of $\rho$ to be the endo-class of any simple character contained in $\rho$. If we write $\boldsymbol{\Theta}$ for this endo-class, then $\rho$ contains a simple R-supertype $\boldsymbol{\lambda}(\alpha) \in \mathcal{T}(\mathrm{G}, \boldsymbol{\Theta}, \mathrm{R})$ for some $\alpha \in \mathrm{X}$ with trivial $\Sigma$-stabilizer.

Remark 3.8. - The positive integer $s(\rho)$ associated with $\rho$ in $\S 3.1$ is the order of the $\Gamma$-stabilizer of $\alpha$.

\section{5.}

We call an inertial class of supercuspidal pairs of G simple if it contains a pair of the form

$$
\left(\mathrm{GL}_{m / r}(\mathrm{D})^{r}, \rho \otimes \cdots \otimes \rho\right)
$$

for some integer $r$ dividing $m$ and some supercuspidal R-representation $\rho$ of $\mathrm{GL}_{m / r}(\mathrm{D})$, and we define the endo-class of such an inertial class to be the endo-class of $\rho$, that is, the endo-class of any simple character contained in $\rho$. By [37, Section 8], there is a bijective correspondence between simple inertial classes of supercuspidal pairs of $\mathrm{G}$ and equivalence classes of simple supertypes of G, that preserves endo-classes. More precisely, the inertial class of (3.6), denoted $\Omega$, corresponds to the equivalence class of a simple supertype $(\mathrm{J}, \lambda)$ if and only if the irreducible representations of $\mathrm{G}$ occurring as a subquotient of the compact induction of $\lambda$ to $\mathrm{G}$ are exactly those irreducible representations of $\mathrm{G}$ occurring as a subquotient of the parabolic induction to G of an element of $\Omega$.

From the previous paragraph, we have an endo-class $\Theta$ and a maximal $\beta$-extension $\kappa$. Combining the map (3.4) with the correspondence between simple inertial classes of supercuspidal pairs 
and equivalence classes of simple supertypes, we get the following result. Given $\alpha \in \mathrm{X}$, we write $\boldsymbol{\Omega}(\alpha)$ for the inertial class of supercuspidal pairs of $\mathrm{G}$ that corresponds to $\boldsymbol{\lambda}(\alpha)$.

Proposition 3.9. - The map

$$
\alpha \mapsto \Omega(\alpha)
$$

induces a surjection from $\mathrm{X}$ to the set of simple inertial classes of supercuspidal pairs of $\mathrm{G}$ with associated endo-class $\boldsymbol{\Theta}$. Its fibers are the $\Gamma$-orbits of $\mathrm{X}$.

Let us recall the following important result from [27, Théorème 8.16]: given an irreducible Rrepresentation $\pi$ of $\mathrm{G}$, there are integers $m_{1}, \ldots, m_{r} \geqslant 1$ such that $m_{1}+\cdots+m_{r}=m$, and supercuspidal irreducible representations $\rho_{1}, \ldots, \rho_{r}$ of $\mathrm{GL}_{m_{1}}(\mathrm{D}), \ldots, \mathrm{GL}_{m_{r}}(\mathrm{D})$ respectively, such that $\pi$ occurs as a subquotient of the induced representation $\rho_{1} \times \cdots \times \rho_{r}$. Moreover, up to renumbering, the supercuspidal representations $\rho_{1}, \ldots, \rho_{r}$ are unique. The conjugacy class of the supercuspidal pair $\left(\mathrm{GL}_{m_{1}}(\mathrm{D}) \times \cdots \times \mathrm{GL}_{m_{r}}(\mathrm{D}), \rho_{1} \otimes \cdots \otimes \rho_{r}\right)$ is called the supercuspidal support of $\pi$.

Let us call an irreducible R-representation of $\mathrm{G}$ simple if the inertial class of its supercuspidal support is simple. For instance, any discrete series R-representation of $\mathrm{G}$ is simple. We define the endo-class of a simple irreducible representation to be that of its supercuspidal support.

Definition 3.10. - Let $\pi$ be a simple irreducible representation of $\mathrm{G}$ with endo-class $\Theta$. The parametrizing class of $\pi$ is the $\Gamma$-orbit of a character $\alpha \in \mathrm{X}$ such that the two following equivalent conditions hold:

(1) the supercuspidal support of $\pi$ belongs to the inertial class $\boldsymbol{\Omega}(\alpha)$;

(2) the representation $\pi$ occurs as a subquotient of the compact induction of $\boldsymbol{\lambda}(\alpha)$ to $\mathrm{G}$.

The parametrizing class of $\pi$ is denoted $\mathrm{X}(\kappa, \pi)$, or simply $\mathrm{X}(\pi)$ if there is no ambiguity on the maximal $\beta$-extension $\kappa$.

Remark 3.11. - Let $\kappa^{\prime}$ be another maximal $\beta$-extension of the simple character $\theta \in \mathcal{C}(\mathfrak{a}, \beta)$ in G. By [33, Théorème 2.28] there is a character $\chi$ of $\boldsymbol{e}^{\times}$such that $\kappa^{\prime}=\kappa \zeta$, where $\zeta$ is the character of $\mathrm{J}$ trivial on $\mathrm{J}^{1}$ that corresponds to the character $\chi \circ \mathrm{N}_{\boldsymbol{d} / \boldsymbol{e}} \circ \operatorname{det}$ of $\mathcal{G}$, where $\mathrm{N}_{\boldsymbol{d} / \boldsymbol{e}}$ is the norm map with respect to $\boldsymbol{d} / \boldsymbol{e}$. Then we have $\alpha^{\prime} \in \mathrm{X}\left(\kappa^{\prime}, \pi\right)$ if and only if $\alpha^{\prime} \mu \in \mathrm{X}(\kappa, \pi)$, where $\mu$ is the character $\chi \circ \mathrm{N}_{\boldsymbol{k} / \boldsymbol{e}}$ of $\boldsymbol{k}^{\times}$.

Remark 3.12. - When $\mathrm{R}$ has characteristic 0, the two equivalent conditions of Definition 3.10 are also equivalent to:

(3) the representation $\pi$ occurs as a quotient of the compact induction of $\boldsymbol{\lambda}(\alpha)$ to $\mathrm{G}$.

Equivalently, the restriction of $\pi$ to $\mathrm{J}_{r}$ contains $\boldsymbol{\lambda}(\alpha)$ as a subrepresentation.

\section{Linked $\ell$-adic representations}

In this section, we fix a prime number $\ell$ different from $p$. We will distinguish between $\ell$-adic and $\bmod \ell$ representations by using a tilde $\sim$ for $\ell$-adic representations. 
4.1.

Let $\tilde{\pi}$ be an irreducible $\ell$-adic representation of $\mathrm{G}$. Fix a representative $(\mathrm{M}, \widetilde{\rho})$ in the inertial class of its cuspidal support, with $\mathrm{M}$ a standard Levi subgroup $\mathrm{GL}_{m_{1}}(\mathrm{D}) \times \cdots \times \mathrm{GL}_{m_{r}}(\mathrm{D})$ and $\widetilde{\rho}$ of the form $\widetilde{\rho}_{1} \otimes \cdots \otimes \widetilde{\rho}_{r}$ where $\widetilde{\rho}_{i}$ is an $\ell$-adic cuspidal irreducible representation of $\mathrm{GL}_{m_{i}}(\mathrm{D})$ for $i \in\{1, \ldots, r\}$, and with $m_{1}+\cdots+m_{r}=m$. Since $\widetilde{\rho}_{i}$ is determined up to an unramified twist, we may assume it is integral (see paragraph 3.2), and fix an irreducible subquotient $\rho_{i}$ of its reduction $\bmod \ell$. By the classification of $\bmod \ell$ irreducible cuspidal representations in terms of supercuspidal representations [27, Théorème 6.14], there are a unique integer $u_{i} \geqslant 1$ dividing $m_{i}$ and a supercuspidal irreducible representation $\tau_{i}$ of degree $u_{i}$ such that the supercuspidal support of $\rho_{i}$ is inertially equivalent to

$$
\left(\mathrm{GL}_{u_{i}}(\mathrm{D}) \times \cdots \times \mathrm{GL}_{u_{i}}(\mathrm{D}), \tau_{i} \otimes \cdots \otimes \tau_{i}\right)
$$

where the factors are repeated $k_{i}$ times, with $m_{i}=k_{i} u_{i}$.

Definition 4.1. - Let $\tilde{\pi}$ be an irreducible $\ell$-adic representation of $\mathrm{G}$ as above. Let us write

$$
\mathrm{L}=\mathrm{GL}_{u_{1}}(\mathrm{D})^{k_{1}} \times \cdots \times \mathrm{GL}_{u_{r}}(\mathrm{D})^{k_{r}}, \quad \tau=\underbrace{\tau_{1} \otimes \cdots \otimes \tau_{1}}_{k_{1} \text { times }} \otimes \cdots \otimes \underbrace{\tau_{r} \otimes \cdots \otimes \tau_{r}}_{k_{r} \text { times }} .
$$

The inertial class in $\mathrm{G}$ of the supercuspidal pair $(\mathrm{L}, \tau)$, denoted $\mathbf{i}_{\ell}(\widetilde{\pi})$, is uniquely determined by the irreducible representation $\tilde{\pi}$. It is called the $\bmod \ell$ inertial supercuspidal support of $\tilde{\pi}$.

Definition 4.2. - Two irreducible $\ell$-adic representations $\widetilde{\pi}_{1}, \widetilde{\pi}_{2}$ of $\mathrm{G}$ are said to belong to the same $\ell$-block if $\mathbf{i}_{\ell}\left(\tilde{\pi}_{1}\right)=\mathbf{i}_{\ell}\left(\tilde{\pi}_{2}\right)$.

An $\ell$-block in the set $\operatorname{Irr}\left(\mathrm{G}, \overline{\mathbf{Q}}_{\ell}\right)$ of all isomorphism classes of irreducible $\ell$-adic representations of $\mathrm{G}$ is an equivalence class for the equivalence relation defined by $\mathbf{i}_{\ell}$.

Let $\tilde{\pi}$ be an irreducible $\ell$-adic representation of $\mathrm{G}$ as above. By definition, $\mathbf{i}_{\ell}(\tilde{\pi})$ depends only on the inertial class of the supercuspidal support of $\tilde{\pi}$. Assume $\tilde{\pi}$ is integral.

Lemma 4.3. - All irreducible subquotients occurring in $\mathbf{r}_{\ell}(\tilde{\pi})$, the reduction mod $\ell$ of $\tilde{\pi}$, have their supercuspidal support in $\mathbf{i}_{\ell}(\tilde{\pi})$.

Proof. - The representation $\tilde{\pi}$ is a subquotient of $\tilde{\rho}_{1} \times \cdots \times \widetilde{\rho}_{r}$. Since $\tilde{\pi}$ is integral, all the $\tilde{\rho}_{i}$ 's are integral and, by Proposition 3.2, for each $i$ there is an integer $a_{i} \geqslant 1$ such that

$$
\mathbf{r}_{\ell}\left(\tilde{\rho}_{i}\right)=\rho_{i}+\rho_{i} \nu+\cdots+\rho_{i} \nu^{a_{i}-1},
$$

where $\nu$ denotes the unramified $\bmod \ell$ character "absolute value of the reduced norm". Thus any irreducible subquotient of $\mathbf{r}_{\ell}(\widetilde{\pi})$ occurs as a subquotient of $\rho_{1} \nu^{i_{1}} \times \cdots \times \rho_{r} \nu^{i_{r}}$ for some integers $i_{1}, \ldots, i_{r} \in \mathbf{N}$. The result now follows by looking at the supercuspidal support of each $\rho_{i}$.

Corollary 4.4. - Any two integral irreducible $\ell$-adic representations of $\mathrm{G}$ whose reductions mod $\ell$ share a common irreducible component belong to the same $\ell$-block. 


\section{2.}

Let $\tilde{\pi}$ be a simple irreducible $\ell$-adic representation of $\mathrm{G}$. There are an integer $r \geqslant 1$ dividing $m$ and a cuspidal irreducible representation $\widetilde{\rho}$ of $\mathrm{G}_{m / r}$ such that the inertial class of its cuspidal support contains

$$
\left(\mathrm{GL}_{m / r}(\mathrm{D})^{r}, \widetilde{\rho} \otimes \cdots \otimes \widetilde{\rho}\right) .
$$

We may assume $\tilde{\rho}$ is integral. We fix an irreducible subquotient $\rho$ of its reduction modulo $\ell$. As in Paragraph 4.1, there are a unique integer $u \geqslant 1$ dividing $m / r$ and a supercuspidal irreducible representation $\tau$ of degree $u$ such that the supercuspidal support of $\rho$ is inertially equivalent to $\left(\mathrm{GL}_{u}(\mathrm{D}) \times \cdots \times \mathrm{GL}_{u}(\mathrm{D}), \tau \otimes \cdots \otimes \tau\right)$, with $m=k u r$. Therefore, the mod $\ell$ inertial supercuspidal support $\mathbf{i}_{\ell}(\tilde{\pi})$ of the $\ell$-adic simple irreducible representation $\tilde{\pi}$ is the inertial class of the pair

$$
\left(\mathrm{GL}_{u}(\mathrm{D})^{k r}, \tau \otimes \cdots \otimes \tau\right) .
$$

In particular, it is simple.

Recall that, according to [27, Théorème 6.11], any supercuspidal irreducible mod $\ell$ representation can be lifted to an $\ell$-adic irreducible representation. The following lemma is an immediate consequence of the definition of the $\bmod \ell$ inertial supercuspidal support.

Lemma 4.5. - Let $\widetilde{\tau}$ be an $\ell$-adic lift of $\tau$. Any simple irreducible $\ell$-adic representation whose cuspidal support is inertially equivalent to

$$
\left(\mathrm{GL}_{u}(\mathrm{D})^{k r}, \widetilde{\tau} \otimes \cdots \otimes \widetilde{\tau}\right)
$$

is in the same $\ell$-block as $\tilde{\pi}$. In particular, the $\ell$-adic discrete series representation $\mathrm{L}(\tilde{\tau}, k r)$ is in the same $\ell$-block as $\tilde{\pi}$.

\section{3 .}

Recall that we have fixed in Section 2 a smooth character $\psi_{\ell}: \mathrm{F} \rightarrow \overline{\mathbf{Q}}_{\ell}^{\times}$, trivial on $\mathfrak{p}$ but not on $\mathcal{O}$. Since $\mathrm{F}$ is the union of the $\mathfrak{p}^{-i}$ for $i \geqslant 1$ and $p$ is invertible in $\overline{\mathbf{Z}}_{\ell}$, it has values in $\overline{\mathbf{Z}}_{\ell}^{\times}$. For any simple stratum $[\mathfrak{a}, \beta]$ in $\mathrm{M}_{m}(\mathrm{D})$, the set of simple $\ell$-adic characters associated with $[\mathfrak{a}, \beta]$ will be defined with respect to $\psi_{\ell}$ (see Paragraph 3.3), whereas the set of $\ell$-modular simple characters associated with $[\mathfrak{a}, \beta]$ will be defined with respect to the reduction $\bmod \ell$ of $\psi_{\ell}$. Reduction $\bmod \ell$ thus induces a bijection between $\ell$-adic and $\ell$-modular simple characters associated with $[\mathfrak{a}, \beta]$. It also induces a bijection between endo-classes of $\ell$-adic and $\ell$-modular simple characters. Thus we will speak of endo-classes of simple characters, without referring to the coefficient field.

Let $\Theta$ be the endo-class of Paragraphs 3.3-3.5. Fix a $\beta$-extension $\widetilde{\kappa}$ of a maximal $\ell$-adic simple character in $\mathrm{G}$ of endo-class $\boldsymbol{\Theta}$, and write $\mathrm{X}_{\ell}$ for the group of $\ell$-adic characters of $\boldsymbol{k}^{\times}$. The map (3.4) gives us a bijection $\tilde{\boldsymbol{\lambda}}_{\ell}$ from $\mathrm{X}_{\ell} / \Gamma$ onto the set of equivalence classes of $\mathcal{T}\left(\mathrm{G}, \boldsymbol{\Theta}, \overline{\mathbf{Q}}_{\ell}\right)$. Also write $Y_{\ell}$ for the group of $\ell$-modular characters of $\boldsymbol{k}^{\times}$, and $\kappa$ for the reduction $\bmod \ell$ of $\widetilde{\kappa}$. This gives us a bijection $\boldsymbol{\lambda}_{\ell}$ from $\mathrm{Y}_{\ell} / \Gamma$ onto the set of equivalence classes of $\mathcal{T}\left(\mathrm{G}, \boldsymbol{\Theta}, \overline{\mathbf{F}}_{\ell}\right)$. These two bijections are compatible in the following sense. 
Proposition 4.6. - Let $\tilde{\pi}$ be a simple irreducible $\ell$-adic representation of $\mathrm{G}$ with endo-class $\boldsymbol{\Theta}$, let $\alpha \in \mathrm{X}_{\ell}(\tilde{\pi})$ and let $\phi \in \mathrm{Y}_{\ell}$ be the reduction $\bmod \ell$ of $\alpha$. Then the inertial class $\mathbf{i}_{\ell}(\tilde{\pi})$ corresponds through (3.5) and (3.7) to the equivalence class of the simple supertype $\boldsymbol{\lambda}_{\ell}(\phi)$.

Proof. - Write the inertial class of the cuspidal support of $\tilde{\pi}$ as in (4.1). Let $r$ be the degree of $\boldsymbol{k}$ over $\boldsymbol{d}[\alpha]$ and $\widetilde{\sigma}_{0}$ be the $\ell$-adic supercuspidal representation of $\mathrm{GL}_{u}(\boldsymbol{k})$ associated to $\alpha$, where $m^{\prime}=r u$. There is a maximal $\beta$-extension $\widetilde{\kappa}_{0}$ of $\mathrm{GL}_{m / r}(\mathrm{D})$ such that $\widetilde{\kappa}_{0} \otimes \widetilde{\sigma}_{0}$ is a maximal simple type contained in $\widetilde{\rho}$. More precisely, with the notation of Paragraph 3.3 and writing $\mathrm{M}_{r}$ for the Levi subgroup $\mathrm{GL}_{m / r}(\mathrm{D}) \times \cdots \times \mathrm{GL}_{m / r}(\mathrm{D}) \subseteq \mathrm{G}$ and $\mathrm{U}_{r}$ for the unipotent radical of the parabolic subgroup made of upper $r \times r$ block triangular matrices of $\mathrm{G}$, the representation of $\mathrm{J}_{r} \cap \mathrm{M}_{r}$ on the $\mathrm{J}_{r} \cap \mathrm{U}_{r}$-invariant subspace of $\widetilde{\kappa}_{r}$ is $\widetilde{\kappa}_{0} \otimes \cdots \otimes \widetilde{\kappa}_{0}$.

Let $\rho$ be an irreducible component of the reduction $\bmod \ell$ of $\tilde{\rho}$. Then $\rho$ contains the maximal simple type $\kappa_{0} \otimes \sigma_{0}$, where $\kappa_{0}$ is the reduction $\bmod \ell$ of $\widetilde{\kappa}_{0}$ and $\sigma_{0}$ is that of $\widetilde{\sigma}_{0}$.

Let $t$ be the degree of $\boldsymbol{k}$ over $\boldsymbol{d}[\phi]$. By [30, Lemme 3.2], if we write $\rho$ in the form $\operatorname{Sp}(\tau, k)$, with $\tau$ supercuspidal (see Proposition 3.1), then $k r=t$ and $\sigma_{0}$ is the unique nondegenerate irreducible subquotient of the induced representation $\sigma_{1} \times \cdots \times \sigma_{1}$, where $\sigma_{1}$ is the supercuspidal mod $\ell$ representation of $\mathrm{GL}_{m^{\prime} / t}(\boldsymbol{d})$ corresponding to $\phi$. Moreover, if $\kappa_{1}$ denotes the maximal $\beta$-extension of $\mathrm{GL}_{m / t}(\mathrm{D})$ such that the representation of $\mathrm{J}_{t} \cap \mathrm{M}_{t}$ on the $\mathrm{J}_{t} \cap \mathrm{U}_{t}$-invariant subspace of $\kappa_{t}$ is $\kappa_{1} \otimes \cdots \otimes \kappa_{1}$, then $\kappa_{1} \otimes \sigma_{1}$ is a maximal simple type contained in $\tau$. The result follows.

We keep in mind the following straightforward but important fact.

Remark 4.7. - Two simple irreducible $\ell$-adic representations of $G$ in the same $\ell$-block have the same endo-class.

The converse does not hold in general, but we have the following result. Given $\alpha \in \mathrm{X}$, write $[\alpha]$ for its $\Gamma$-orbit and $\phi$ for its reduction $\bmod \ell$. The orbit $[\phi]$ depends only on $[\alpha]$, and is called the reduction $\bmod \ell$ of $[\alpha]$.

Proposition 4.8. - Two simple irreducible $\ell$-adic representations of $\mathrm{G}$ of endo-class $\Theta$ are in the same $\ell$-block if and only if their parametrizing classes have the same reduction mod $\ell$.

Proof. - This follows from Propositions 3.5 and 4.6.

\section{Linked complex representations}

5.1.

We fix a prime number $\ell$ different from $p$ and an isomorphism of fields $\iota_{\ell}: \mathbf{C} \simeq \overline{\mathbf{Q}}_{\ell}$. If $\pi$ is a complex representation of $\mathrm{G}$, write $\iota_{\ell}^{*} \pi$ for the $\ell$-adic representation of $\mathrm{G}$ obtained by extending scalars from $\mathbf{C}$ to $\overline{\mathbf{Q}}_{\ell}$ along $\iota_{\ell}$.

Definition 5.1. - Two irreducible complex representations $\pi_{1}, \pi_{2}$ of $\mathrm{G}$ are said to be $\ell$-linked if the irreducible $\ell$-adic representations $\iota_{\ell}^{*} \pi_{1}$ and $\iota_{\ell}^{*} \pi_{2}$ are in the same $\ell$-block.

Lemma 5.2. - This definition does not depend on the choice of $\iota_{\ell}$. 
Proof. - It is enough to prove that, for any field automorphism $\theta \in \operatorname{Aut}\left(\overline{\mathbf{Q}}_{\ell}\right)$, two simple $\ell$-adic representations $\tilde{\pi}_{1}$ and $\widetilde{\pi}_{2}$ of $\mathrm{G}$ are in the same $\ell$-block if and only if $\tilde{\pi}_{1}^{\theta}$ and $\widetilde{\pi}_{2}^{\theta}$ are in the same $\ell$-block.

Given an irreducible $\ell$-adic representation $\tilde{\pi}$, let $(\mathrm{L}, \tau)$ be an element of its mod $\ell$ inertial supercuspidal support as in Definition 4.1. Then the $\bmod \ell$ inertial supercuspidal support of the irreducible representation $\widetilde{\pi}^{\theta}$ is the inertial class of $\left(\mathrm{L}, \tau^{\theta}\right)$. The result follows.

\section{2 .}

Recall that we have fixed in Section 2 a smooth character $\psi: \mathrm{F} \rightarrow \mathbf{C}^{\times}$, trivial on $\mathfrak{p}$ but not on $\mathcal{O}$. For any simple stratum $[\mathfrak{a}, \beta]$, the set of simple complex characters associated with $[\mathfrak{a}, \beta]$ will be defined with respect to this choice (see Paragraphs 3.3 and 4.3). We may and will assume that the character $\iota_{\ell} \circ \psi$ is the character $\psi_{\ell}$ of Paragraph 4.3. This gives us a bijection between endo-classes of complex and $\ell$-adic simple characters of G. Again, we will speak of endo-classes of simple characters, without referring to the coefficient field.

Let $\kappa$ be a $\beta$-extension of some maximal complex simple character in $\mathrm{G}$ having endo-class $\Theta$. Write $\mathrm{X}$ for the group of complex characters of $\boldsymbol{k}^{\times}$.

Lemma 5.3. - Let $\pi$ be a simple irreducible complex representation of $\mathrm{G}$ with endo-class $\boldsymbol{\Theta}$. Then we have

$$
\alpha \in \mathrm{X}(\kappa, \pi) \quad \Leftrightarrow \quad \iota_{\ell} \circ \alpha \in \mathrm{X}_{\ell}\left(\iota_{\ell}^{*} \kappa, \iota_{\ell}^{*} \pi\right) .
$$

Proof. - We have $\alpha \in \mathrm{X}(\kappa, \pi)$ if and only if $\pi$ contains the simple type $\boldsymbol{\lambda}(\alpha)=\boldsymbol{\kappa}(\alpha) \otimes \boldsymbol{\sigma}(\alpha)$, which occurs if and only if $\iota_{\ell}^{*} \pi$ contains the $\ell$-adic simple type $\iota_{\ell}^{*} \lambda(\alpha)$. Thus it suffices to prove that $\iota_{\ell}^{*} \boldsymbol{\lambda}(\alpha)$ is equal to $\tilde{\boldsymbol{\lambda}}_{\ell}\left(\iota_{\ell} \circ \alpha\right)$, where $\tilde{\boldsymbol{\lambda}}_{\ell}$ is the map as in Paragraph 4.3 defined with respect to the maximal $\beta$-extension $\iota_{\ell}^{*} \kappa$.

Firstly, the $\ell$-adic $\beta$-extension $\widetilde{\boldsymbol{\kappa}}_{\ell}\left(\iota_{\ell} \circ \alpha\right)$ associated with $\iota_{\ell} \circ \alpha$ with respect to $\iota_{\ell}^{*} \kappa$ is equal to $\iota_{\ell}^{*} \boldsymbol{\kappa}(\alpha)$. Secondly, the $\ell$-adic supercuspidal representation $\tilde{\boldsymbol{\sigma}}_{\ell}\left(\iota_{\ell} \circ \alpha\right)$ associated with $\iota_{\ell} \circ \alpha$ (with respect to the choice of an isomorphism (3.3)) is equal to $\iota_{\ell}^{*} \sigma(\alpha)$, since it is characterized by a trace formula (see Remark 3.4). The result follows.

Definition 5.4. - Let $\alpha \in \mathrm{X}$. The $\ell$-regular part of $\alpha$ is the unique complex character $\alpha_{\ell} \in \mathrm{X}$ whose order is prime to $\ell$ and such that $\alpha \alpha_{\ell}^{-1}$ has order a power of $\ell$.

Given $\alpha \in \mathrm{X}$, the orbit $\left[\alpha_{\ell}\right]$ depends only on $[\alpha]$. It is called the $\ell$-regular part of $[\alpha]$, denoted $[\alpha]_{\ell}$.

Proposition 5.5. - Two simple irreducible complex representations of $\mathrm{G}$ with endo-class $\boldsymbol{\Theta}$ are $\ell$-linked if and only if the $\ell$-regular parts of their parametrizing classes are equal.

Proof. - Let $\pi_{1}, \pi_{2}$ be simple irreducible complex representations of $\mathrm{G}$ with endo-class $\boldsymbol{\Theta}$. We fix $\alpha_{i} \in \mathrm{X}\left(\kappa, \pi_{i}\right)$ for each $i=1,2$. By Lemma 5.3 and Proposition 4.8, the representations $\pi_{1}, \pi_{2}$ are $\ell$-linked if and only if $\left[\iota_{\ell} \circ \alpha_{1}\right]$ and $\left[\iota_{\ell} \circ \alpha_{2}\right]$ have the same reduction $\bmod \ell$. But the reduction $\bmod \ell$ of $\left[\iota_{\ell} \circ \alpha\right]$, for a character $\alpha \in \mathrm{X}$, is the same as that of $\left[\iota_{\ell} \circ \alpha_{\ell}\right]$. It follows that we have $\left[\iota_{\ell} \circ\left(\alpha_{1}\right)_{\ell}\right]=\left[\iota_{\ell} \circ\left(\alpha_{2}\right)_{\ell}\right]$, thus $\left[\alpha_{1}\right]_{\ell}=\left[\alpha_{2}\right]_{\ell}$. 
5.3.

Recall that $q$ is the cardinality of the residue field of $F$. For each prime number $\ell$ dividing

$$
\left(q^{n}-1\right)\left(q^{n-1}-1\right) \ldots(q-1)
$$

we fix an isomorphism of fields $\iota_{\ell}: \mathbf{C} \simeq \overline{\mathbf{Q}}_{\ell}$.

Definition 5.6. - Two irreducible complex representations $\pi, \pi^{\prime}$ of $\mathrm{G}$ are linked if there are a finite family $\ell_{1}, \ldots, \ell_{r}$ of prime numbers dividing (5.1) and a finite family of irreducible complex representations $\pi=\pi_{0}, \pi_{1}, \ldots, \pi_{r}=\pi^{\prime}$ such that, for all integers $i \in\{1, \ldots, r\}$, the representations $\pi_{i-1}$ and $\pi_{i}$ are $\ell_{i}$-linked.

Remark 5.7. - By Lemma 5.2, this does not depend on the choice of the isomorphisms $\iota_{\ell}$ for $\ell$ dividing (5.1).

Two linked simple complex representations of $\mathrm{G}$ have the same endo-class (see Remark 4.7). The converse is given by the following proposition.

Proposition 5.8. - Two simple irreducible complex representations are linked if and only if they have the same endo-class.

Proof. - Assume $\pi$ and $\pi^{\prime}$ are simple irreducible complex representations with the same endoclass $\Theta$. Let $\alpha$ and $\alpha^{\prime}$ be characters in $\mathrm{X}(\pi)$ and $\mathrm{X}\left(\pi^{\prime}\right)$, respectively, and write $\xi=\alpha^{\prime} \alpha^{-1}$. Let $\ell_{1}, \ldots, \ell_{r}$ be the prime numbers dividing (5.1). The character $\xi$ decomposes uniquely as

$$
\xi=\xi_{1} \ldots \xi_{r}
$$

where the order of $\xi_{i}$ is a power of $\ell_{i}$, for $i \in\{1, \ldots, r\}$. Write $\alpha_{0}=\alpha$ and define inductively

$$
\alpha_{i}=\alpha_{i-1} \cdot \xi_{i}
$$

for all $i \in\{1, \ldots, r\}$. Let $\pi_{i}$ be a simple irreducible complex representation of endo-class $\Theta$ and parametrizing class $\left[\alpha_{i}\right]$. The result follows from Proposition 5.5.

Remark 5.9. - Suppose that $\pi$ and $\pi^{\prime}$ are discrete series representations with the same endoclass. The proof of Proposition 5.8 shows that the simple representations $\pi_{1}, \ldots, \pi_{r-1} \operatorname{linking} \pi$ to $\pi^{\prime}$ can be chosen to be discrete series representations as well.

\section{4 .}

Let $\pi$ be an irreducible complex representation of G. Fix a representative (M, $\rho)$ in its cuspidal support, with $\mathrm{M}=\mathrm{GL}_{m_{1}}(\mathrm{D}) \times \cdots \times \mathrm{GL}_{m_{r}}(\mathrm{D})$ and $\rho=\rho_{1} \otimes \cdots \otimes \rho_{r}$, with $m_{1}+\cdots+m_{r}=m$, and where $\rho_{i}$ is a cuspidal irreducible representation of $\mathrm{GL}_{m_{i}}(\mathrm{D})$ for $i \in\{1, \ldots, r\}$. Write $\boldsymbol{\Theta}_{i}$ for the endo-class of $\rho_{i}$ and $g_{i}$ for the degree of $\boldsymbol{\Theta}_{i}$. We define the semi-simple endo-class of $\pi$ to be the formal sum

$$
\boldsymbol{\Theta}(\pi)=\sum_{i=1}^{r} \frac{m_{i} d}{g_{i}} \cdot \boldsymbol{\Theta}_{i}
$$

in the free abelian semigroup generated by all F-endo-classes. It depends only on the inertial class of the cuspidal support of $\pi$. 
Note that, if $\pi$ is a simple irreducible representation with endo-class $\boldsymbol{\Theta}$, then its semi-simple endo-class is $\boldsymbol{\Theta}(\pi)=(n / g) \cdot \boldsymbol{\Theta}$ where $g$ is the degree of $\boldsymbol{\Theta}$.

The following theorem, which is our first main result, generalizes Proposition 5.8.

Theorem 5.10. - Two irreducible complex representations are linked if and only if they have the same semi-simple endo-class.

Proof. - Any two linked irreducible complex representations automatically have the same semisimple endo-class. We thus start with two irreducible complex representations $\pi, \pi^{\prime}$ with the same semi-simple endo-class. By [28, Théorème 4.16], the representation $\pi$ can be written

$$
\pi=\pi_{1} \times \pi_{2} \times \cdots \times \pi_{k}
$$

where $\pi_{1}, \pi_{2}, \ldots, \pi_{k}$ are simple irreducible representations whose inertial cuspidal supports are pairwise distinct, and this decomposition is unique up to renumbering. We have the following straightforward lemma.

Lemma 5.11. - Let $\delta$ be an irreducible complex representation of $\mathrm{GL}_{m-k}(\mathrm{D})$ for some integer $k \in\{1, \ldots, m-1\}$. Let $\sigma, \sigma^{\prime}$ be two irreducible complex representations of $\mathrm{GL}_{k}(\mathrm{D})$, and let $\pi, \pi^{\prime}$ be irreducible subquotients of $\sigma \times \delta$ and $\sigma^{\prime} \times \delta$, respectively. If $\sigma$ and $\sigma^{\prime}$ are linked, then $\pi$ and $\pi^{\prime}$ are linked.

For each $i \in\{1, \ldots, k\}$, thanks to Lemma 5.11 and Proposition 5.8, we may and will assume that $\pi_{i}$ is a discrete series representation of the form $\mathrm{L}\left(\rho_{i}, r_{i}\right)$ for some cuspidal representation $\rho_{i}$ of $\mathrm{GL}_{m_{i}}(\mathrm{D})$ with same endo-class as $\pi_{i}$ and some integer $r_{i}$, such that $m_{1} r_{1}+\cdots+m_{k} r_{k}=m$. We may even assume that $\rho_{i}$ has minimal degree among all cuspidal irreducible representations of $\mathrm{GL}_{a}(\mathrm{D}), a \geqslant 1$, with the same endo-class as $\pi_{i}$. This amounts to saying that $m_{i}$ is equal to $g_{i} /\left(g_{i}, d\right)$, where $g_{i}$ is the degree of the endo-class of $\pi_{i}$.

Moreover, if $\rho_{i}$ and $\rho_{j}$ have the same endo-class for some $i, j \in\{1, \ldots, k\}$, then they have the same degree, thus they are linked. We thus may assume $\rho_{1}, \ldots, \rho_{k}$ have distinct endo-classes, denoted $\boldsymbol{\Theta}_{1}, \ldots, \boldsymbol{\Theta}_{k}$, respectively.

Similarly, we may assume the representation $\pi^{\prime}$ decomposes as a product $\pi_{1}^{\prime} \times \pi_{2}^{\prime} \times \cdots \times \pi_{t}^{\prime}$, where $\pi_{j}^{\prime}$ is a discrete series representation of the form $\mathrm{L}\left(\rho_{j}^{\prime}, s_{j}\right)$ for some cuspidal representation $\rho_{j}^{\prime}$ of $\mathrm{GL}_{m_{j}^{\prime}}(\mathrm{D})$ and some integer $s_{j} \geqslant 1$, and we may assume that the endo-classes $\boldsymbol{\Theta}_{1}^{\prime}, \ldots, \boldsymbol{\Theta}_{t}^{\prime}$ of $\rho_{1}^{\prime}, \ldots, \rho_{t}^{\prime}$ are distinct. It follows that $k=t$ and, up to renumbering, we may assume that we have $\boldsymbol{\Theta}_{i}^{\prime}=\boldsymbol{\Theta}_{i}$ for each $i \in\{1, \ldots, k\}$. It then follows that $\rho_{i}^{\prime}$ and $\rho_{i}$ have the same degree, by minimality of $m_{i}$.

Since $\pi$ and $\pi^{\prime}$ have the same semi-simple endo-class, we have $s_{i}=r_{i}$ for all $i$, thus $\pi_{i}$ and $\pi_{i}^{\prime}$ have the same degree. Proposition 5.8 then implies that $\pi_{i}$ and $\pi_{i}^{\prime}$ are linked. Theorem 5.10 now follows from Lemma 5.11 again.

\section{Application to the local Jacquet-Langlands correspondence}

We fix $n=m d$ and write $\mathrm{G}=\mathrm{GL}_{m}(\mathrm{D})$ and $\mathrm{H}=\mathrm{GL}_{n}(\mathrm{~F})$. As in the introduction, we write $\mathcal{D}(\mathrm{G}, \mathbf{C})$ for the set of all isomorphism classes of complex discrete series representations of $\mathrm{G}$, 
and similarly for $\mathrm{H}$. We write

$$
\boldsymbol{\pi}: \mathcal{D}(\mathrm{G}, \mathbf{C}) \rightarrow \mathcal{D}(\mathrm{H}, \mathbf{C})
$$

for the local Jacquet-Langlands correspondence.

\section{1 .}

We fix an isomorphism of fields $\iota_{\ell}: \mathbf{C} \simeq \overline{\mathbf{Q}}_{\ell}$ and write (as in $[\mathbf{3 0}]$ )

$$
\tilde{\boldsymbol{\pi}}_{\ell}: \mathcal{D}\left(\mathrm{G}, \overline{\mathbf{Q}}_{\ell}\right) \rightarrow \mathcal{D}\left(\mathrm{H}, \overline{\mathbf{Q}}_{\ell}\right)
$$

for the $\ell$-adic local Jacquet-Langlands correspondence between $\ell$-adic discrete series representations of $\mathrm{G}$ and $\mathrm{H}$. The correspondence (6.2) does not depend on the choice of $\iota_{\ell}$ ([30, Remarque 10.1]). According to [2, Paragraph 3.1], there is a unique surjective group homomorphism

$$
\tilde{\mathbf{J}}_{\ell}: \mathcal{R}\left(\mathrm{H}, \overline{\mathbf{Q}}_{\ell}\right) \rightarrow \mathcal{R}\left(\mathrm{G}, \overline{\mathbf{Q}}_{\ell}\right)
$$

where $\mathcal{R}\left(\mathrm{G}, \overline{\mathbf{Q}}_{\ell}\right)$ is the Grothendieck group of finite length $\ell$-adic representations of $\mathrm{G}$, with the following property: given positive integers $n_{1}, \ldots, n_{r}$ such that $n_{1}+\cdots+n_{r}=n$ and an $\ell$-adic discrete series representation $\widetilde{\sigma}_{i}$ of $\mathrm{GL}_{n_{i}}(\mathrm{~F})$ for each $i$, the image of the product $\widetilde{\sigma}_{1} \times \cdots \times \widetilde{\sigma}_{r}$ by $\tilde{\mathbf{J}}_{\ell}$ is 0 if $n_{i}$ is not divisible by $d$ for at least one $i$, and is $\tilde{\pi}_{1} \times \cdots \times \widetilde{\pi}_{r}$ otherwise, where $n_{i}=m_{i} d$ and $\tilde{\pi}_{i}$ is the $\ell$-adic discrete series representation of $\mathrm{GL}_{m_{i}}(\mathrm{D})$ whose Jacquet-Langlands transfer is $\tilde{\sigma}_{i}$, for each $i$.

By [30, Théorème 12.4], there exists a unique surjective group homomorphism of Grothendieck groups $\mathbf{J}_{\ell}: \mathcal{R}\left(\mathrm{H}, \overline{\mathbf{F}}_{\ell}\right) \rightarrow \mathcal{R}\left(\mathrm{G}, \overline{\mathbf{F}}_{\ell}\right)$ such that the diagram

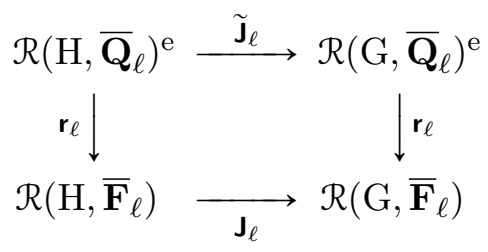

is commutative, where $\mathcal{R}\left(\mathrm{G}, \overline{\mathbf{Q}}_{\ell}\right)^{\mathrm{e}}$ is the subgroup of $\mathcal{R}\left(\mathrm{G}, \overline{\mathbf{Q}}_{\ell}\right)$ generated by integral irreducible representations, and $\mathcal{R}\left(\mathrm{G}, \overline{\mathbf{F}}_{\ell}\right)$ is the Grothendieck group of $\ell$-modular representations of $\mathrm{G}$.

Proposition 6.1. - Let $\tilde{\pi}_{1}$ and $\tilde{\pi}_{2}$ be $\ell$-adic discrete series representations of $\mathrm{G}$, and write $\widetilde{\sigma}_{1}$, $\tilde{\sigma}_{2}$ for their Jacquet-Langlands transfers to $\mathrm{H}$, respectively. If $\widetilde{\sigma}_{1}, \widetilde{\sigma}_{2}$ are in the same $\ell$-block of $\mathrm{H}$, then $\tilde{\pi}_{1}, \tilde{\pi}_{2}$ are in the same $\ell$-block of $\mathrm{G}$.

Proof. - Let us write $\tilde{\sigma}_{i}=\mathrm{L}\left(\tilde{\rho}_{i}, r_{i}\right)$ and $k_{i}=k\left(\widetilde{\rho}_{i}\right)$ for $i=1,2$. Then $k_{1} r_{1}=k_{2} r_{2}$, which we denote by $v$, and the $\bmod \ell$ inertial supercuspidal support of $\widetilde{\sigma}_{1}$ and $\widetilde{\sigma}_{2}$ contains the supercuspidal pair

$$
\left(\mathrm{GL}_{u}(\mathrm{~F}) \times \cdots \times \mathrm{GL}_{u}(\mathrm{~F}), \tau \otimes \cdots \otimes \tau\right),
$$

with $u v=m$ and for some mod $\ell$ supercuspidal representation $\tau$ of $\mathrm{GL}_{u}(\mathrm{D})$. Fix an $\ell$-adic lift $\widetilde{\tau}$ of $\tau$ and write $\widetilde{\sigma}=\mathrm{L}(\widetilde{\tau}, v)$. The representation $\widetilde{\sigma}$ is in the same $\ell$-block as $\widetilde{\sigma}_{1}, \widetilde{\sigma}_{2}$, by Lemma 4.5. If we write $\tilde{\pi}$ for the $\ell$-adic discrete series representation of $\mathrm{G}$ whose transfer to $\mathrm{H}$ is $\tilde{\sigma}$, then it is enough to prove that $\tilde{\pi}$ is in the same $\ell$-block as $\tilde{\pi}_{1}$. 
In the remainder of the proof, it will be more convenient for us to deal with Speh representations rather than discrete series representations, as in [30]. We thus apply the Zelevinski involution to $\tilde{\pi}, \widetilde{\pi}_{1}$ and $\widetilde{\sigma}, \widetilde{\sigma}_{1}$ and thus get $\ell$-adic Speh representations.

Let us write $\widetilde{\sigma}^{*}$ for the Zelevinski dual of $\widetilde{\sigma}$. Its reduction $\bmod \ell$ is the $\ell$-modular super-Speh representation $\mathrm{Z}(\tau, v)$, by $\left[\mathbf{2 7}\right.$, Théorème 9.39]. If we write $\widetilde{\pi}^{*}=\mathrm{Z}(\widetilde{\alpha}, t)$ for the Zelevinski dual of $\widetilde{\pi}$, for some $t$ dividing $m$ and some cuspidal irreducible representation $\widetilde{\alpha}$ of $\mathrm{GL}_{m / t}(\mathrm{D})$, then its reduction $\bmod \ell$ contains the Speh representation $\mathrm{Z}(\alpha, t)$ where $\alpha$ is an irreducible component of the reduction $\bmod \ell$ of $\widetilde{\alpha}$ (see for instance [30, Proposition 1.10]). The cuspidal representation $\alpha$ need not be supercuspidal but, according to Proposition 3.1, it can be written as $\operatorname{Sp}(\beta, k)$ for $k=k(\alpha)$ and some supercuspidal irreducible representation $\beta$.

We now look at the reduction mod $\ell$ of the Zelevinski dual of $\widetilde{\sigma}_{1}$. It is $\mathrm{Z}\left(\rho_{1}, r_{1}\right)$ where $\rho_{1}$, the reduction $\bmod \ell$ of $\widetilde{\rho}_{1}$, can be written as $\operatorname{Sp}\left(\tau \chi, k_{1}\right)$ for some unramified character $\chi$. By twisting $\tilde{\pi}_{1}$ by an unramified character of $\mathrm{G}$, we may assume that $\chi$ is trivial. According to $[\mathbf{2 7}$, Lemme 9.41], the representation $\mathrm{Z}\left(\rho_{1}, k_{1}\right)$ decomposes as a $\mathbf{Z}$-linear combination of products of the form

$$
\mathrm{Z}\left(\tau \nu^{i_{1}}, v_{1}\right) \times \cdots \times \mathrm{Z}\left(\tau \nu^{i_{r}}, v_{r}\right)
$$

with $v_{1}+\cdots+v_{r}=v$ and $i_{1}, \ldots, i_{r} \in \mathbf{Z}$, where $\nu$ stands for the absolute value of the reduced norm, as usual. (For an explicit formula for this decomposition, see [30, Sections 11 and 12].) Thanks to the commutative diagram above, the reduction modulo $\ell$ of the Zelevinski dual of $\widetilde{\pi}_{1}$ will be made of products of the form

$$
\mathrm{Z}\left(\alpha \nu^{i_{1}}, t_{1}\right) \times \cdots \times \mathrm{Z}\left(\alpha \nu^{i_{r}}, t_{r}\right)
$$

with $t_{1}+\cdots+t_{r}=t$ and $i_{1}, \ldots, i_{r} \in \mathbf{Z}$, all of whose irreducible subquotients have supercuspidal support inertially equivalent to $\left(\mathrm{GL}_{w}(\mathrm{D}) \times \cdots \times \mathrm{GL}_{w}(\mathrm{D}), \beta \otimes \cdots \otimes \beta\right)$, with $w k t=m$. The result follows from Corollary 4.4.

\section{2 .}

Proposition 6.1 implies that two complex discrete series representations $\pi_{1}, \pi_{2}$ of $\mathrm{G}$ are linked if their Jacquet-Langlands transfers are linked. We have the following refinement.

Proposition 6.2. - Let $\widetilde{\pi}_{1}$ and $\tilde{\pi}_{2}$ be $\ell$-adic discrete series representations of $\mathrm{G}$, and write $\widetilde{\sigma}_{1}$, $\tilde{\sigma}_{2}$ for their Jacquet-Langlands transfers to $\mathrm{H}$, respectively. Then $\widetilde{\sigma}_{1}, \widetilde{\sigma}_{2}$ are in the same $\ell$-block of $\mathrm{H}$ if and only if $\tilde{\pi}_{1}, \tilde{\pi}_{2}$ are in the same $\ell$-block of $\mathrm{G}$.

Proof. - Proposition 6.1 implies that the $\ell$-adic Jacquet-Langlands correspondence (6.2) induces a well-defined map from $\ell$-blocks of discrete series representations of $\mathrm{H}$ to those of G: given an $\ell$-block of $\mathrm{H}$, if $\widetilde{\sigma}$ is any $\ell$-adic discrete series representation in that block, then the $\ell$-block of the transfer to $\mathrm{G}$ of $\widetilde{\sigma}$ is independent of the choice of $\widetilde{\sigma}$. This map also preserves depth so that, for any non-negative rational number $r \in \mathbf{Q}_{+}$, we get a well-defined map from $\ell$-blocks of discrete series representations of depth $r$ of $\mathrm{H}$ to $\ell$-blocks of discrete series representations of depth $r$ of G. This map is between two finite sets of the same cardinality, since they are parametrized by the same objects: an endo-class of depth $r$ and, by Proposition 4.8, a parametrizing class upto reduction $\bmod \ell$. It is clearly surjective, so is also injective. 
Allowing $\ell$ to vary, we deduce

Theorem 6.3. - Two complex discrete series representations of $\mathrm{G}$ are linked if and only if their transfers to $\mathrm{H}$ are linked.

It follows that Proposition 5.8 (together with Remark 5.9) induces a map

$$
\pi_{1}: \mathcal{E}_{n}(\mathrm{~F}) \rightarrow \mathcal{E}_{n}(\mathrm{~F})
$$

depending on $\mathrm{G}$, where $\mathcal{E}_{n}(\mathrm{~F})$ is the set of F-endo-classes of degree dividing $n$. More precisely, given an endo-class $\Theta \in \mathcal{E}_{n}(\mathrm{~F})$ and a complex discrete series representation $\pi$ of $\mathrm{G}$ of endo-class $\Theta$, the endo-class of the Jacquet-Langlands transfer of $\pi$ to $\mathrm{H}$ depends only on $\Theta$ : we denote it $\boldsymbol{\pi}_{1}(\boldsymbol{\Theta})$. This map does not depend on the choice of the isomorphisms $\iota_{\ell}$ for $\ell$ dividing (5.1).

Proposition 6.4. - The map $\boldsymbol{\pi}_{1}$ is bijective.

Proof. - This map is clearly surjective: given an endo-class $\boldsymbol{\Theta} \in \mathcal{E}_{n}(\mathrm{~F})$, and any discrete series representation $\sigma \in \mathcal{D}(\mathrm{H}, \mathbf{C})$ with endo-class $\boldsymbol{\Theta}$, the endo-class of its inverse Jacquet-Langlands transfer $\boldsymbol{\pi}^{-1}(\sigma) \in \mathcal{D}(\mathrm{G}, \mathbf{C})$ is an antecedent of $\boldsymbol{\Theta}$ by $\boldsymbol{\pi}_{1}$.

Now let $\pi, \pi^{\prime} \in \mathcal{D}(\mathrm{G}, \mathbf{C})$ have Jacquet-Langlands transfers $\sigma, \sigma^{\prime}$ to $\mathrm{H}$ with the same endo-class. By Proposition 5.8 and Remark 5.9, the representations $\sigma, \sigma^{\prime}$ are linked by a family of discrete series representations. By Theorem 6.3, the same holds for $\pi$ and $\pi^{\prime}$. Thus they have the same endo-class.

Recall that the parametric degree of a cuspidal representation of $\mathrm{G}$ has been defined in $§ 3.1$.

Proposition 6.5. - For every complex discrete series representation of $\mathrm{G}$, there is a cuspidal complex representation of $\mathrm{G}$ with the same endo-class and with parametric degree $n$.

Proof. - Let $\pi$ be a complex discrete series representation of G with endo-class $\Theta$. To find a complex cuspidal representation with same endo-class and parametric degree $n$, we need to find a $\operatorname{Gal}(\boldsymbol{k} / \boldsymbol{d})$-regular complex character $\alpha \in \mathrm{X}$ which is also $\operatorname{Gal}(\boldsymbol{k} / \boldsymbol{e})$-regular. The latter implies the former, so let us find a $\operatorname{Gal}(\boldsymbol{k} / \boldsymbol{e})$-regular character $\alpha \in \mathrm{X}$. For this, it is enough to choose for $\alpha$ a generator of the cyclic group $\mathrm{X}$.

As an immediate consequence, we see that, given an endo-class $\Theta$ in $\mathcal{E}_{n}(\mathrm{~F})$, if there is a single complex cuspidal representation $\rho$ of $\mathrm{G}$ with endo-class $\Theta$ and parametric degree $n$ such that $\boldsymbol{\pi}(\rho)$ has endo-class $\boldsymbol{\Theta}$, then $\boldsymbol{\pi}_{1}(\boldsymbol{\Theta})$ is equal to $\boldsymbol{\Theta}$.

\section{3 .}

In this paragraph, the division algebra $\mathrm{D}$ is fixed, but we allow the positive integer $m$ to vary. Given an $m \geqslant 1$, we write $\boldsymbol{\pi}_{1, m}$ for the map (6.3) induced by the Jacquet-Langlands correspondence from $\mathcal{D}\left(\mathrm{GL}_{m}(\mathrm{D}), \mathbf{C}\right)$ to $\mathcal{D}\left(\mathrm{GL}_{m d}(\mathrm{~F}), \mathbf{C}\right)$. Recall (see Lemma 3.3) that, associated with an endo-class $\Theta \in \mathcal{E}(\mathrm{F})$, there is an integer called its ramification index. 
Theorem 6.6. - (1) There is a unique map

$$
\boldsymbol{j}=\boldsymbol{j}_{\mathrm{D}}: \mathcal{E}(\mathrm{F}) \rightarrow \mathcal{E}(\mathrm{F}),
$$

depending only on $\mathrm{D}$, such that, for any integer $m \geqslant 1$, the restriction of $\boldsymbol{j}$ to $\mathcal{E}_{m d}(\mathrm{~F})$ coincides with the map $\boldsymbol{\pi}_{1, m}$.

(2) The map $\boldsymbol{j}$ is bijective, and it is the identity on all essentially tame endo-classes (that is, all endo-classes whose ramification index is prime to $p$ ).

Proof. - Uniqueness follows from the fact that $\mathcal{E}(\mathrm{F})$ is the union of the $\mathcal{E}_{m d}(\mathrm{~F})$, for $m \geqslant 1$.

In order to prove the existence of $\boldsymbol{j}$, it suffices to prove that for all $m, k \geqslant 1$, the maps $\boldsymbol{\pi}_{1, m}$ and $\boldsymbol{\pi}_{1, k}$ coincide on $\varepsilon_{m d}(\mathrm{~F}) \cap \mathcal{E}_{k d}(\mathrm{~F})=\mathcal{E}_{r d}(\mathrm{~F})$, where $r$ denotes the greatest common divisor of $m, k$. For this, let $\boldsymbol{\Theta} \in \mathcal{E}_{r d}(\mathrm{~F})$, and $\rho$ be a cuspidal irreducible representation of $\mathrm{GL}_{r}(\mathrm{D})$ with endo-class $\Theta$ and parametric degree $r d$. Its Jacquet-Langlands transfer to $\mathrm{GL}_{r d}(\mathrm{~F})$ is a cuspidal representation denoted $\sigma$, whose endo-class is denoted $\Theta^{\prime}$. Then, for any $a \geqslant 1$, the discrete series representation $\mathrm{L}(\rho, a)$ of $\mathrm{GL}_{a r}(\mathrm{D})$ has endo-class $\boldsymbol{\Theta}$, and its transfer $\mathrm{L}(\sigma, a)$ to $\mathrm{GL}_{a r d}(\mathrm{~F})$ has endo-class $\boldsymbol{\Theta}^{\prime}$. It follows that $\boldsymbol{\pi}_{1, m}(\boldsymbol{\Theta})=\boldsymbol{\pi}_{1, r}(\boldsymbol{\Theta})=\boldsymbol{\Theta}^{\prime}$. The bijectivity of $\boldsymbol{j}$ follows from the fact that all the maps $\boldsymbol{\pi}_{1, m}$, for $m \geqslant 1$, are bijective.

To prove the second part of (2), given an essentially tame endo-class $\boldsymbol{\Theta}$, it suffices to find a single complex cuspidal representation $\rho$ of $\mathrm{G}$ with endo-class $\Theta$ and parametric degree $n$ such that $\boldsymbol{\pi}(\rho)$ has endo-class $\boldsymbol{\Theta}$. But it follows from [13] - which gives an explicit, type-theoretic description of the Jacquet-Langlands transfer of complex cuspidal representations of $\mathrm{G}$ with essentially tame endo-class and parametric degree $n$ - that this is true of any complex cuspidal representation $\rho$ of $\mathrm{G}$ with endo-class $\boldsymbol{\Theta}$ and parametric degree $n$.

Remark 6.7. - After this paper was written, Dotto proved the Endo-class Invariance Conjecture in $[\mathbf{2 0}]$. Thus it is now known that the map $\boldsymbol{j}$ of Theorem 6.6 is in fact the identity.

\section{Reduction to the maximal totally ramified case}

We continue with the previous notation, so that $\mathrm{G}=\mathrm{GL}_{m}(\mathrm{D})$ and $\mathrm{H}=\mathrm{GL}_{n}(\mathrm{~F})$. In this section, we closely follow the ideas of $[\mathbf{1 3}$, Section 6] to make a further reduction to the maximal totally ramified case (see Paragraph 1.1). All representations in this section are complex.

\section{1 .}

Let $\pi$ be a cuspidal (complex) representation of $\mathrm{G}$ with parametric degree $n$. Let $(\mathbf{J}, \boldsymbol{\lambda})$ be an extended maximal simple type of $\mathrm{G}$ contained in $\pi[\mathbf{2 8}, \S 3.1$ and Théorème 3.11], attached to a simple stratum $[\mathfrak{a}, \beta]$ and a simple character $\theta \in \mathfrak{C}(\mathfrak{a}, \beta)$. Write $\mathrm{B}$ for the centralizer of $\beta$ in $\mathrm{M}_{m}(\mathrm{D})$, so that $\mathrm{B} \simeq \mathrm{M}_{m^{\prime}}\left(\mathrm{D}^{\prime}\right)$, for some integer $m^{\prime} \geqslant 1$ and $\mathrm{F}[\beta]$-division algebra $\mathrm{D}^{\prime}$. Fix a maximal unramified extension $\mathrm{L}$ of $\mathrm{F}[\beta]$ in $\mathrm{B}$, and write $\mathrm{K}$ for the maximal unramified subextension of L over F.

We fix a root of unity $\zeta \in \mathrm{K}$ of order relatively prime to $p$ such that $\mathrm{K}=\mathrm{F}[\zeta]$. Write $\mathrm{G}_{\mathrm{K}}$ for the centralizer of $\mathrm{K}$ in $\mathrm{G}$. Let $u$ be a pro-unipotent, elliptic regular element of $\mathrm{G}_{\mathrm{K}}$ in the sense 
of [13, Paragraph 1.6]. The element $h=\zeta u$ then lies in the set $\mathrm{G}_{\mathrm{reg}}^{\text {ell }}$ of elliptic regular elements of $\mathrm{G}$, so we have

$$
\operatorname{tr} \pi(h)=\sum_{x \in \mathrm{G} / \mathbf{J}} \operatorname{tr} \boldsymbol{\lambda}\left(x^{-1} h x\right)
$$

as in $[\mathbf{1 3},(6.3 .1)]$. Write $\mathbf{J}=\mathbf{J}(\mathfrak{a}, \beta)=\mathbf{J} \cap \mathfrak{a}^{\times}$. A coset $x \mathbf{J}$ can only contribute to the sum if we have $x^{-1} h x \in \mathbf{J}$ or, equivalently, $x^{-1} h x \in \mathrm{J}$. As in [13, 6.3 Lemma], such a coset $x \mathbf{J}$ is contained in $\mathrm{N}_{\mathrm{G}}(\mathrm{K}) \mathbf{J}$, where $\mathrm{N}_{\mathrm{G}}(\mathrm{K})$ is the normalizer of $\mathrm{K}$ in $\mathrm{G}$.

Write $\Psi$ for the Galois group of $\mathrm{K} / \mathrm{F}$ and $\Gamma$ for that of $\mathrm{L} / \mathrm{F}[\beta]$. Restriction of operators identifies $\Gamma$ with a subgroup of $\Psi$. Write $\Psi_{\mathrm{t}}$ for the unique subgroup of $\Gamma$ (thus of $\Psi$ ) of order $m^{\prime} s(\pi)$, where $s(\pi)$ is the integer introduced in Paragraph 3.1. Observe, thanks to the description of the group $\mathbf{J}$ in $[\mathbf{3 4}, 5.1]$, that $\Psi_{\mathrm{t}}$ is the image of $\mathbf{J} \cap \mathrm{N}_{\mathrm{G}}(\mathrm{K})$ under the surjective map $\mathrm{N}_{\mathrm{G}}(\mathrm{K}) / \mathrm{G}_{\mathrm{K}} \rightarrow \Psi$. As in $[\mathbf{1 3},(6.3 .2)]$, we have

$$
\operatorname{tr} \pi(\zeta u)=\sum_{\alpha \in \Psi / \Psi_{\mathrm{t}}} \sum_{y \in \mathrm{G}_{\mathrm{K}} / \mathbf{J}_{\mathrm{K}}} \operatorname{tr} \boldsymbol{\lambda}\left(y^{-1} \zeta^{\alpha} u^{\alpha} y\right)
$$

where $\mathbf{J}_{\mathrm{K}}=\mathbf{J} \cap \mathrm{G}_{\mathrm{K}}$.

Let us fix a uniformizer $\varpi_{F}$ of $F$. We choose an irreducible representation $\boldsymbol{\kappa}$ of $\mathbf{J}$ such that:

(1) the restriction of $\boldsymbol{\kappa}$ to $\mathrm{J}$ is a $\beta$-extension of $\theta$;

(2) the character $\operatorname{det}(\boldsymbol{\kappa})$ has order a power of $p$;

(3) the automorphism $\kappa\left(\varpi_{\mathrm{F}}\right)$ is the identity.

Note that such a representation is not unique. We now write

$$
\boldsymbol{\sigma}=\operatorname{Hom}_{\mathrm{J}^{1}}(\boldsymbol{\kappa}, \boldsymbol{\lambda})
$$

which carries an action of $\mathbf{J}$ given by $g \cdot f=\boldsymbol{\lambda}(g) \circ f \circ \boldsymbol{\kappa}(g)^{-1}$ for $g \in \mathbf{J}$ and $f \in \boldsymbol{\sigma}$. This representation is irreducible and trivial on $\mathrm{J}^{1}=\mathrm{J}^{1}(\mathfrak{a}, \beta)$, and we have the decomposition $\boldsymbol{\lambda}=\boldsymbol{\kappa} \otimes \boldsymbol{\sigma}$. As in $[\mathbf{1 3},(6.4 .1)]$ this gives us

$$
\operatorname{tr} \pi(\zeta u)=\sum_{\alpha \in \Psi / \Psi_{\mathrm{t}}} \operatorname{tr} \boldsymbol{\sigma}\left(\zeta^{\alpha}\right) \sum_{y \in \mathrm{G}_{\mathrm{K}} / \mathbf{J}_{\mathrm{K}}} \operatorname{tr} \boldsymbol{\kappa}\left(y^{-1} \zeta^{\alpha} u^{\alpha} y\right) .
$$

We are now going to interpret the sum over $\mathrm{G}_{\mathrm{K}} / \mathbf{J}_{\mathrm{K}}$ as the trace of a cuspidal irreducible representation of $\mathrm{G}_{\mathrm{K}}$.

\section{2 .}

Write $\theta_{\mathrm{K}}$ for the restriction of $\theta$ to $\mathrm{H}^{1}(\mathfrak{a}, \beta) \cap \mathrm{G}_{\mathrm{K}}$, which is the interior $\mathrm{K} / \mathrm{F}$-lift of the simple character $\theta$ in the sense of [6, Section 5]. The group $\mathbf{J}_{\mathrm{K}}$ is also the normalizer of $\theta_{\mathrm{K}}$ in $\mathrm{G}_{\mathrm{K}}$. We choose an irreducible representation $\boldsymbol{\kappa}_{\mathrm{K}}$ of $\mathbf{J}_{\mathrm{K}}$ such that:

(1) the restriction of $\boldsymbol{\kappa}_{\mathrm{K}}$ to $\mathrm{J}_{\mathrm{K}}$ is a $\beta$-extension of $\theta_{\mathrm{K}}$;

(2) the character $\operatorname{det}\left(\boldsymbol{\kappa}_{\mathrm{K}}\right)$ has order a power of $p$;

(3) the automorphism $\boldsymbol{\kappa}_{\mathrm{K}}\left(\varpi_{\mathrm{F}}\right)$ is the identity. 
Again, such a choice may not be unique. The pair $\left(\mathbf{J}_{\mathrm{K}}, \boldsymbol{\kappa}_{\mathrm{K}}\right)$ is an extended maximal simple type in $\mathrm{G}_{\mathrm{K}}$. It thus defines a cuspidal irreducible representation $\rho$ of $\mathrm{G}_{\mathrm{K}}$. By [12, (3.4.3) and (5.6.2)], there is a sign $\epsilon \in\{-1,+1\}$ such that

$$
\operatorname{tr} \boldsymbol{\kappa}\left(y^{-1} \zeta^{\alpha} u^{\alpha} y\right)=\epsilon \cdot \operatorname{tr} \boldsymbol{\kappa}_{\mathrm{K}}\left(y^{-1} \zeta^{\alpha} u^{\alpha} y\right) .
$$

As in $[\mathbf{1 3},(6.4 .2)]$ this gives us

$$
\operatorname{tr} \pi(\zeta u)=\epsilon \sum_{\alpha \in \Psi / \Psi_{\mathrm{t}}} \operatorname{tr} \boldsymbol{\sigma}\left(\zeta^{\alpha}\right) \operatorname{tr} \rho^{\alpha^{-1}}(u)
$$

We do not know whether a result similar to [13,6.5 Lemma] holds, that is, we do not know whether the $\Psi$-stabilizers of $\rho$ and of its inertial class are both equal to $\Gamma$. However, let $\Psi_{0}$ denote the stabilizer in $\Psi$ of the inertial class of $\rho$ and let $\mathrm{X}_{0}$ be a set of representatives for $\Psi \bmod \Psi_{0}$. For $\gamma \in \Psi_{0}$ there is an unramified character $\chi_{\gamma}$ of $\mathrm{G}_{\mathrm{K}}$ such that $\rho^{\gamma^{-1}} \simeq \rho \chi_{\gamma}$. Since $u$ is pro-unipotent (thus compact) we have $\chi_{\gamma}^{\alpha^{-1}}(u)=1$, for all $\alpha \in \Psi / \Psi_{\mathrm{t}}$. Therefore (7.1) can be rewritten as

$$
\operatorname{tr} \pi(\zeta u)=\epsilon \sum_{\alpha \in \mathrm{X}_{0}} \operatorname{tr} \rho^{\alpha^{-1}}(u) \sum_{\gamma \in \Psi_{0} / \Psi_{\mathrm{t}}} \operatorname{tr} \sigma\left(\zeta^{\alpha \gamma}\right)
$$

Note that the map

$$
w: \zeta \mapsto \sum_{\gamma \in \Psi_{0} / \Psi_{t}} \operatorname{tr} \sigma\left(\zeta^{\gamma}\right)
$$

is not identically zero on the set of K/F-regular roots of unity, by [38, Theorem 1.1(ii)]. Thus there is an $\alpha \in \Psi$ such that the coefficient $w\left(\zeta^{\alpha}\right)$ in (7.2) is nonzero.

\section{3.}

Now write $\pi^{\prime}$ for the Jacquet-Langlands transfer of $\pi$ to H. Since $\pi$ has parametric degree $n$, the torsion number $t(\pi)$ is equal to the degree of $\mathrm{K}$ over $\mathrm{F}$. We now do for $\pi^{\prime}$ what we did for $\pi$.

Let $\left(\mathbf{J}^{\prime}, \boldsymbol{\lambda}^{\prime}\right)$ be an extended maximal simple type of $\mathrm{H}$ contained in $\pi^{\prime}$, attached to a simple stratum $\left[\mathfrak{a}^{\prime}, \beta^{\prime}\right]$. Write $\mathrm{B}^{\prime}$ for the centralizer of $\beta^{\prime}$ in $\mathrm{M}_{n}(\mathrm{~F})$, fix a maximal unramified extension $\mathrm{L}^{\prime}$ of $\mathrm{F}\left[\beta^{\prime}\right]$ in $\mathrm{B}^{\prime}$ and write $\mathrm{K}^{\prime}$ for the maximal unramified subextension of $\mathrm{L}^{\prime}$ over $\mathrm{F}$. The relation $t(\pi)=t\left(\pi^{\prime}\right)$, together with the fact that $\pi^{\prime}$ also has parametric degree $n$, implies that $\mathrm{K}^{\prime}$ and $\mathrm{K}$ have the same degree over F. Therefore, we may identify the maximal unramified subextension of $\mathrm{L}^{\prime} / \mathrm{F}$ with $\mathrm{K}$.

We have an analogue $\boldsymbol{\sigma}^{\prime}$ of $\boldsymbol{\sigma}$ and an analogue $\rho^{\prime}$ of $\rho$ in the argument of the previous paragraph so that we get

$$
\operatorname{tr} \pi^{\prime}\left(\zeta u^{\prime}\right)=\epsilon^{\prime} \sum_{\alpha^{\prime} \in \mathrm{X}_{0}^{\prime}} \operatorname{tr} \rho^{\alpha^{\prime-1}}\left(u^{\prime}\right) \sum_{\gamma^{\prime} \in \Psi_{0}^{\prime} / \Psi_{\mathrm{t}}^{\prime}} \operatorname{tr} \boldsymbol{\sigma}^{\prime}\left(\zeta^{\alpha^{\prime} \gamma^{\prime}}\right)
$$

where $\zeta \in \mathrm{K}$ is as above, $u^{\prime}$ is a pro-unipotent elliptic regular element of the centralizer $\mathrm{H}_{\mathrm{K}}$ of $\mathrm{K}$ in $\mathrm{H}, \epsilon^{\prime} \in\{-1,+1\}$ is a sign and the subgroups $\Psi_{\mathrm{t}}^{\prime}, \Psi_{0}^{\prime}$ and $\mathrm{X}_{0}^{\prime}$ are defined as in the previous paragraph. If $\zeta u^{\prime}$ is chosen to have the same reduced characteristic polynomial over $\mathrm{F}$ as $\zeta u$, this is 
equal to $(-1)^{n-m} \cdot \operatorname{tr} \pi(\zeta u)$, by the trace relation characterizing the Jacquet-Langlands correspondence. We thus get:

$$
\epsilon^{\prime} \sum_{\alpha^{\prime} \in \mathrm{X}_{0}^{\prime}} w^{\prime}\left(\zeta^{\alpha^{\prime}}\right) \operatorname{tr} \rho^{\prime \alpha^{\prime-1}}\left(u^{\prime}\right)=(-1)^{n-m} \cdot \epsilon \sum_{\alpha \in \mathrm{X}_{0}} w\left(\zeta^{\alpha}\right) \operatorname{tr} \rho^{\alpha^{-1}}(u)
$$

where the function $w$ and its analogue $w^{\prime}$ are defined by (7.3).

We apply $[\mathbf{1 3}, 6.6 \mathrm{Lemma}]$ (note that $\rho$ has maximal parametric degree since $\mathrm{L} / \mathrm{K}$ is maximal). The $\rho^{\prime \alpha^{\prime-1}}, \alpha^{\prime} \in \mathrm{X}_{0}^{\prime}$, are not unramified twists of each other, and the same holds for the JacquetLanglands transfers to $\mathrm{H}_{\mathrm{K}}$ of the $\rho^{\alpha^{-1}}, \alpha \in \mathrm{X}_{0}$. Thanks to the linear independence of characters, it follows that there is an $\alpha \in \Psi$ such that

$$
\boldsymbol{\pi}_{\mathrm{K}}\left(\rho^{\alpha^{-1}}\right)=\rho^{\prime} \chi
$$

for some unramified character $\chi$ of $\mathrm{H}_{\mathrm{K}}$, where $\boldsymbol{\pi}_{\mathrm{K}}$ is the local Jacquet-Langlands correspondence from $\mathrm{G}_{\mathrm{K}}$ to $\mathrm{H}_{\mathrm{K}}$.

Assume now that $\boldsymbol{\pi}_{\mathrm{K}}$ preserves K-endo-classes for maximal totally ramified cuspidal representations of $\mathrm{G}_{\mathrm{K}}$. Write $\mathcal{E}(\mathrm{F})$ for the set of endo-classes over $\mathrm{F}$, and likewise $\mathcal{E}(\mathrm{K})$. The representations $\rho^{\alpha^{-1}}$ and $\rho^{\prime}$ have the same endo-class in $\mathcal{E}(\mathrm{K})$. But the K-endo-class of $\rho^{\alpha^{-1}}$ (respectively, of $\rho^{\prime}$ ) is a K/F-lift of the F-endo-class of $\pi$ (respectively, of $\pi^{\prime}$ ) in the sense of [8, Definition 9.7]. It follows (for instance by applying the restriction map of [8, Corollary 9.13] from $\mathcal{E}(\mathrm{K})$ to $\mathcal{E}(\mathrm{F})$ ) that $\pi, \pi^{\prime}$ have the same F-endo-class. Thus we have proved Theorem A of the introduction:

Theorem 7.1. - Assume that, for all $\mathrm{F}$ and $n$, and all maximal totally ramified, cuspidal irreducible complex representations $\rho$ of $\mathrm{G}$, the representations $\rho$ and $\boldsymbol{\pi}(\rho)$ have the same endo-class. Then the map $\boldsymbol{\pi}_{1}$ is the identity.

\section{Explicit Jacquet-Langlands correspondence up to unramified twist}

Now let us fix an endo-class $\boldsymbol{\Theta} \in \mathcal{E}_{n}(\mathrm{~F})$, and suppose that $\boldsymbol{\pi}_{1}(\boldsymbol{\Theta})=\boldsymbol{\Theta}$. Write $\mathcal{D}_{0}(\mathrm{G}, \boldsymbol{\Theta})$ for the set of inertial classes of discrete series representations of $G$ with endo-class $\Theta$. The local Jacquet-Langlands correspondence (6.1) thus induces a bijective map

$$
\pi_{0}: \mathcal{D}_{0}(\mathrm{G}, \boldsymbol{\Theta}) \rightarrow \mathcal{D}_{0}(\mathrm{H}, \boldsymbol{\Theta}) .
$$

The cuspidal support induces a bijection between $\mathcal{D}_{0}(\mathrm{G}, \boldsymbol{\Theta})$ and the set of inertial classes of simple supercuspidal pairs of $\mathrm{G}$ with endo-class $\Theta$.

\section{1.}

We fix a simple stratum $[\mathfrak{a}, \beta]$ in $\mathrm{M}_{m}(\mathrm{D})$ such that $\mathfrak{b}=\mathfrak{a} \cap \mathrm{B}$ is maximal in $\mathrm{B}$, together with a simple character $\theta \in \mathcal{C}(\mathfrak{a}, \beta)$ with endo-class $\boldsymbol{\Theta}$, and a $\beta$-extension $\kappa$ of $\theta$. The integer $m^{\prime}$ coming from (3.3) is $m^{\prime}=m(d, g) / g$, where $g$ denotes the degree of $\boldsymbol{\Theta}$. Write $\mathrm{X}$ for the group of complex characters of $\boldsymbol{k}^{\times}$. Thanks to Proposition 3.5 (see also (3.7)) we have a bijective map

$$
\begin{aligned}
\mathrm{X} / \Gamma & \rightarrow \mathcal{D}_{0}(\mathrm{G}, \boldsymbol{\Theta}) \\
{[\alpha] } & \mapsto \Omega(\kappa, \alpha)
\end{aligned}
$$


where $\Omega(\kappa, \alpha)$ is the inertial class of discrete series representations of $\mathrm{G}$ that contain the simple type $\boldsymbol{\lambda}(\alpha)$.

Similarly, we choose a maximal simple character $\theta^{\prime} \in \mathcal{C}\left(\mathfrak{a}^{\prime}, \beta^{\prime}\right)$ in $\mathrm{H}$ with endo-class $\Theta$ together with a maximal $\beta$-extension $\kappa^{\prime}$ of $\theta^{\prime}$. We fix a finite extension $\boldsymbol{k}^{\prime}$ of the residue field $\boldsymbol{e}^{\prime}$ of $\mathrm{F}\left[\beta^{\prime}\right]$ of degree $n^{\prime}=n / g$, which gives us a parameter set $\mathrm{X}^{\prime} / \Gamma^{\prime}$. We thus get a bijection $\left[\alpha^{\prime}\right] \mapsto \Omega\left(\kappa^{\prime}, \alpha^{\prime}\right)$ between $\mathrm{X}^{\prime} / \Gamma^{\prime}$ and $\mathcal{D}_{0}(\mathrm{H}, \boldsymbol{\Theta})$, similar to $(8.2)$.

Let us fix an isomorphism of $\boldsymbol{f}$-extensions $\boldsymbol{e} \simeq \boldsymbol{e}^{\prime}$, where $\boldsymbol{f}$ denotes the residue field of $\mathrm{F}$. We thus may assume that $\boldsymbol{k}^{\prime}=\boldsymbol{k}$, which identifies the parameter sets $\mathrm{X}^{\prime} / \Gamma^{\prime}$ and $\mathrm{X} / \Gamma$. Let $\Upsilon$ be the unique bijective map such that the diagram

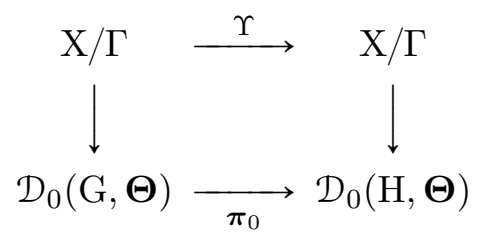

is commutative, where the vertical maps are given by (8.2) and its analogue for $\mathrm{H}$. It depends on the choice of the maximal $\beta$-extensions $\kappa$ and $\kappa^{\prime}$, as well as the $\boldsymbol{f}$-isomorphism $\boldsymbol{e} \simeq \boldsymbol{e}^{\prime}$ (see Remark 3.7 for the dependency in $\boldsymbol{k}$ ). We would like to describe $\Upsilon$. The purpose of Proposition 8.8 below is to show that, in a certain sense, by considering various $m \geqslant 1$ such that $m d$ is divisible by the degree of $\boldsymbol{\Theta}$, one can reduce the computation of $\Upsilon([\alpha])$ to the case where $\alpha$ is suitably regular.

By Proposition 5.5 and Corollary 6.2, we have the following fact.

Proposition 8.1. - For any prime number $\ell$, the bijection $\Upsilon$ is compatible with taking $\ell$-regular parts. More precisely, the $\Gamma$-orbits of $\alpha, \beta \in \mathrm{X}$ have the same $\ell$-regular part if and only if the $\Gamma$-orbits $\Upsilon([\alpha])$ and $\Upsilon([\beta])$ have the same $\ell$-regular part.

Proposition 8.1 suggests that, with a suitable choice of $\ell$, it may be possible to deduce $\Upsilon([\alpha])$ from the knowledge of $\Upsilon([\beta])$. We will illustrate this idea in Proposition 8.8 below.

\section{2.}

We first give another property of the map $\Upsilon$. Set $n^{\prime}=n / g=m^{\prime} d^{\prime}$. Given $\alpha \in \mathrm{X}$, let $f$ be the cardinality of its $\Gamma$-orbit, and write

$$
s(\alpha)=s_{\mathrm{D}}([\alpha])=\frac{d^{\prime}}{\left(f, d^{\prime}\right)} .
$$

Recall that $d^{\prime}$ is the degree of $\boldsymbol{d}$ over $\boldsymbol{e}$ (the residue field of $\mathrm{F}[\beta]$ ), thus $d^{\prime}=d /(d, g)$. Note that the cardinality of its $\operatorname{Gal}(\boldsymbol{k} / \boldsymbol{d})$-orbit is equal to $f /\left(f, d^{\prime}\right)$, which was denoted by $u$ in Paragraph 3.3.

Definition 8.2. - We call the integer $f$ the parametric degree of $\alpha \in \mathrm{X}$.

This is related to the notion of parametric degree for a discrete series representation as follows: any discrete series representation in $\Omega(\kappa, \alpha)$ has parametric degree $f g$.

Since the local Jacquet-Langlands correspondence preserves the parametric degree (see [13]) we have the following result.

Lemma 8.3. - For all $\alpha \in \mathrm{X}$, the parametric degrees of $[\alpha]$ and $\Upsilon([\alpha])$ are equal. 
Note that $\Omega(\kappa, \alpha)$ is made of cuspidal representations with cuspidal Jacquet-Langlands transfers if and only if $f=n^{\prime}$, that is, if and only if $\alpha$ is $\boldsymbol{e}$-regular. Indeed, from [13], a discrete series representation of $\mathrm{G}$ is cuspidal with cuspidal Jacquet-Langlands transfer if and only if its parametric degree is $n$.

\section{3.}

Let $a \geqslant 1$ be a positive integer. We consider the simple stratum $\left[\mathfrak{a}^{*}, \beta\right]$ in $\mathrm{M}_{a m}(\mathrm{D})$, where $\mathfrak{a}^{*}$ is the hereditary order $\mathrm{M}_{a}(\mathfrak{a})$, and write $\theta^{*} \in \mathcal{C}\left(\mathfrak{a}^{*}, \beta\right)$ for the transfer of $\theta$ in the sense of [32, 3.3.3]. Associated with $\kappa$, there is a coherent choice of a maximal $\beta$-extension $\kappa^{*}$ of the simple character $\theta^{*}\left(\left[\mathbf{2 8}\right.\right.$, Remarque 5.17]). We fix a finite extension $\boldsymbol{k}^{*}$ of $\boldsymbol{k}$ of degree $a$. Write $\mathrm{X}^{*}$ for the group of complex characters of $\boldsymbol{k}^{* \times}$ and $\Gamma^{*}$ for the Galois group of $\boldsymbol{k}^{*} / \boldsymbol{e}$. Repeating the arguments of Paragraph 8.1 with $\mathrm{GL}_{a m}(\mathrm{D})$ and $\mathrm{GL}_{a n}(\mathrm{~F})$, we get a bijective map $\Upsilon^{*}: \mathrm{X}^{*} / \Gamma^{*} \rightarrow \mathrm{X}^{*} / \Gamma^{*}$. We have the following straightforward result.

Lemma 8.4. - Let $[\alpha] \in \mathrm{X} / \Gamma$, and let $\mathrm{L}(\rho, r)$ be in the inertial class $\Omega(\kappa, \alpha)$, for some integer $r$ dividing $m$ and some irreducible cuspidal representation $\rho$ of $\mathrm{GL}_{m / r}(\mathrm{D})$. Then $\mathrm{L}(\rho$, ar $)$ is in the inertial class $\Omega\left(\kappa^{*}, \alpha^{*}\right)$, where $\alpha^{*}$ is the character $\alpha \circ \mathrm{N}_{\boldsymbol{k}^{*} / \boldsymbol{k}}$ of $\boldsymbol{k}^{* \times}$.

Proof. - With the notation of Paragraph 3.3 and writing $\mathrm{M}$ for the Levi subgroup $\mathrm{G} \times \cdots \times \mathrm{G} \subseteq$ $\mathrm{GL}_{a m}(\mathrm{D})$ and $\mathrm{U}$ for the unipotent radical of the parabolic subgroup made of upper $a \times a$ block triangular matrices of $\mathrm{GL}_{a m}(\mathrm{D})$, this follows from the fact that the representation of $\mathrm{J}\left(\mathfrak{a}_{r}^{*}, \beta\right) \cap \mathrm{M}$ on the $\mathrm{J}\left(\mathfrak{a}_{r}^{*}, \beta\right) \cap \mathrm{U}$-invariant subspace of the transfer $\kappa_{a r}^{*}$ of $\kappa^{*}$ to $\mathrm{J}\left(\mathfrak{a}_{a r}^{*}, \beta\right)$ is $\kappa \otimes \cdots \otimes \kappa$.

For $\alpha \in \mathrm{X}$, the orbit $\left[\alpha^{*}\right]$ depends only on $[\alpha]$, and we denote it $[\alpha]^{*}$. By Lemma 8.4 we thus have

$$
\Upsilon^{*}\left(\left[\alpha^{*}\right]\right)=\Upsilon(\alpha)^{*}
$$

for any character $\alpha \in \mathrm{X}$.

Given $\alpha \in \mathrm{X}$, we write $f$ for its parametric degree, and $\boldsymbol{e}[\alpha]$ for the subfield of $\boldsymbol{k}$ of degree $f$ over $e$.

Lemma 8.5. - Let $\alpha \in \mathrm{X}$. There are an integer $a \geqslant 1$, a prime number $\ell \neq p$ not dividing the order of $\boldsymbol{e}[\alpha]^{\times}$and an $\boldsymbol{e}$-regular character $\beta \in \mathrm{X}^{*}$ such that $\beta \equiv \alpha^{*} \bmod \ell$.

Proof. - First recall the following result, known as Zsigmondy's Theorem [45].

Lemma 8.6. - Let $b, r \geqslant 2$ be integers. There exists a prime number $\ell$ which divides $b^{r}-1$ but not $b^{i}-1$ for any $i \in\{1, \ldots, r-1\}$, except when $r=6$ and $b=2$, and when $r=2$ and $b=2^{k}-1$ for some $k \geqslant 1$.

Let us write $\mathrm{Q}$ for the cardinality of $\boldsymbol{e}$, and let us fix an $a \geqslant 1$ such that $a n^{\prime}>6 f$. Applying Lemma 8.6 with $b=\mathrm{Q}^{f}$ and $r=a n^{\prime} / f$, we obtain a prime number $\ell$ dividing $b^{r}-1$ but not dividing $b^{i}-1$ for any $i \in\{1, \ldots, r-1\}$. It follows that $b$ has order $r$ in the group $(\mathbf{Z} / \ell \mathbf{Z})^{\times}$.

Let $\xi$ be a nontrivial character of $\boldsymbol{k}^{* \times}$ of order $\ell$. Then the character $\beta=\xi \alpha^{*}$ is congruent to $\alpha^{*} \bmod \ell$. Since the order of $\alpha$ is prime to $\ell$ (for it divides $b-1$ ), the cardinality of the $\Gamma$-orbit 
of $\beta$ is the least common multiple of $f$ and the order of $\mathrm{Q}$ in $(\mathbf{Z} / \ell \mathbf{Z})^{\times}$. This cardinality is equal to $f r=a n^{\prime}$, thus $\beta$ is $\boldsymbol{e}$-regular.

Remark 8.7. - (1) The choice $a=1$ is not always possible. For instance, if $\alpha$ is trivial, $\boldsymbol{e}$ has 7 elements and $n^{\prime}=2$, then no prime number $\ell$ satisfies the required condition. We thank Guy Henniart for a suggestion that brought us to introduce the process described here.

(2) The proof of Lemma 8.5 shows that, for any character $\alpha \in \mathrm{X}$, we can choose $a$ to be any integer $\geqslant 7$. Moreover, the choice of $a$ and $\ell$ only depend on the parametric degree $f$, not on $\alpha$.

(3) Note that $\ell$ cannot be 2 . Indeed we have $\ell \neq p$ and, if $p$ is odd, then the fact that $\ell$ does not divide $\mathrm{Q}^{f}-1$ (the order of $\boldsymbol{e}[\alpha]^{\times}$) implies that $\ell \neq 2$.

With the notation of Lemma 8.5, we get the following result.

Proposition 8.8. - Assume that $\Upsilon^{*}([\beta])=[\beta \mu]$ for some character $\mu \in \mathrm{X}^{*}$. Then $\mu_{\ell}=\nu^{*}$ for some character $\nu \in \mathrm{X}$ and we have $\Upsilon([\alpha])=[\alpha \nu]$.

Proof. - Let us write $\Upsilon([\alpha])=\left[\alpha^{\prime}\right]$ for some $\alpha^{\prime} \in \mathrm{X}$. Then $\left[\alpha^{\prime *}\right] \equiv[\beta \mu] \bmod \ell$. By Lemma 8.3, the parametric degree of $\alpha^{\prime}$ is $f$, thus $\boldsymbol{e}\left[\alpha^{\prime}\right]=\boldsymbol{e}[\alpha]$. It follows that $\ell$ does not divide the order of $\alpha^{\prime}$. Write $\beta=\xi \alpha^{*}$ for some character $\xi$ whose order is a power of $\ell$. Taking $\ell$-regular parts, we get $\left[\alpha^{\prime *}\right]_{\ell}=\left[\alpha^{\prime *}\right]=\left[\alpha^{*} \mu_{\ell}\right]$. Changing $\alpha^{\prime}$ in its $\Gamma$-orbit, we may assume that $\alpha^{\prime *}=\alpha^{*} \mu_{\ell}$. Thus $\mu_{\ell}=\nu^{*}$ for some $\nu \in \mathrm{X}$. Since $\mathrm{N}_{\boldsymbol{k}^{*} / \boldsymbol{k}}$ is surjective, we get $\Upsilon([\alpha])=[\alpha \nu]$.

\section{The essentially tame case}

The purpose of this section is to illustrate Proposition 8.8 in the essentially tame case. Assume that $\boldsymbol{\Theta}$ is essentially tame: we thus have $\boldsymbol{\pi}_{1}(\boldsymbol{\Theta})=\boldsymbol{\Theta}$ by Corollary 6.6. As in Paragraph 8.1, we will fix maximal simple characters $\theta, \theta^{\prime}$ in $\mathrm{G}, \mathrm{H}$ with endo-class $\boldsymbol{\Theta}$, but we must be careful here: for our purpose, these choices have to be compatible, in a sense that we define in Paragraph 9.1.

That we need to take care of this compatibility was brought to our attention by the work of Dotto $[\mathbf{2 0}]$, who resolves this rigidity problem in essentially the same way, though with a slightly different language.

Recall (Corollary 6.6 and Lemma 3.3) that the tameness assumption on $\Theta$ means that $\mathrm{F}[\beta]$ is tamely ramified over $\mathrm{F}$, for any simple stratum $[\mathfrak{a}, \beta]$ and any simple character $\theta \in \mathcal{C}(\mathfrak{a}, \beta)$ of endo-class $\boldsymbol{\Theta}$. We will see other properties of essentially tame endo-classes below. We also refer the reader to $[\mathbf{1 1}$, Section 1] for more details.

As in Section 8, we write $g$ for the degree of $\Theta$ and set $n^{\prime}=n / g$.

\section{1 .}

In order to formulate our Compatibility Assumption below, it is convenient to use the notion of ps-character defined in [6, Definition 1.5]. Fix once and for all a separable closure $\overline{\mathrm{F}}$ of $\mathrm{F}$. Its residue field $\overline{\boldsymbol{f}}$ is an algebraic closure of the residue field $\boldsymbol{f}$ of $\mathrm{F}$. Let $\boldsymbol{k}$ be the unique extension of $\boldsymbol{f}$ of degree $n^{\prime}$ contained in $\overline{\boldsymbol{f}}$ and write $\mathrm{X}$ for the group of complex characters of $\boldsymbol{k}^{\times}$. 
Let us fix a ps-character $(\Theta, 0, \beta)$ of endo-class $\Theta$ with $\beta \in \overline{\mathrm{F}}$. Write $\mathrm{E}$ for the field $\mathrm{F}[\beta]$ and $\boldsymbol{e}$ for its residue field, which canonically identifies with an extension of $\boldsymbol{f}$ contained in $\boldsymbol{k}$. Write $\Gamma$ for the Galois group of $\boldsymbol{k}$ over $\boldsymbol{e}$. Since $\boldsymbol{\Theta}$ is essentially tame, $\mathrm{E}$ is tamely ramified over F.

We now fix a homomorphism $\iota: \mathrm{E} \rightarrow \mathrm{M}_{m}(\mathrm{D})$ of $\mathrm{F}$-algebras and a principal order $\mathfrak{a}$ in $\mathrm{M}_{m}(\mathrm{D})$ normalized by $\iota \mathrm{E}^{\times}$, such that the intersection $\mathfrak{b}$ of $\mathfrak{a}$ with the centralizer $\mathrm{B}$ of $\iota \mathrm{E}$ in $\mathrm{M}_{m}(\mathrm{D})$ is a maximal order. By $\left[\boldsymbol{6}\right.$, Definition 1.5], this gives us a maximal simple stratum $[\mathfrak{a}, \iota \beta]$ in $\mathrm{M}_{m}(\mathrm{D})$ and a maximal simple character $\theta \in \mathcal{C}(\mathfrak{a}, \iota \beta)$ of endo-class $\boldsymbol{\Theta}$. This also defines an $\boldsymbol{f}$-isomorphism

$$
\phi_{\iota}: e \rightarrow e_{\iota}
$$

where $\boldsymbol{e}_{\iota}$ denotes the residue field of $\iota$ E. As in Paragraph 3.3, we fix an extension $\boldsymbol{k}_{\iota}$ of $\boldsymbol{e}_{\iota}$. Write $\mathrm{X}_{\iota}$ for the group of complex characters of $\left(\boldsymbol{k}_{\iota}\right)^{\times}$and $\Gamma_{\iota}$ for the Galois group of $\boldsymbol{k}_{\iota}$ over $\boldsymbol{e}_{\iota}$. The $\boldsymbol{f}$-isomorphism $\phi_{\iota}$ allows us to identify $\mathrm{X} / \Gamma$ and $\mathrm{X}_{\iota} / \Gamma_{\iota}$. Write $\kappa$ for the unique $\beta$-extension of $\theta$ whose determinant has order a power of $p$. This choice gives us a bijective map between $\mathrm{X}_{\iota} / \Gamma_{\iota}$ and $\mathcal{D}_{0}(\mathrm{G}, \boldsymbol{\Theta})$ as in (8.2). Composing with the identification above, we get a bijection

$$
\mathrm{X} / \Gamma \rightarrow \mathcal{D}_{0}(\mathrm{G}, \boldsymbol{\Theta})
$$

denoted $\boldsymbol{\omega}$, depending on the various choices we have made.

Using the same ps-character $(\Theta, 0, \beta)$ as above, we now make similar choices for H: an F-homomorphism $\iota^{\prime}: \mathrm{E} \rightarrow \mathrm{M}_{n}(\mathrm{~F})$ and a principal order $\mathfrak{a}^{\prime}$. This gives us a maximal simple character $\theta^{\prime}$, which is the transfer of $\theta$ in the sense of [32, Paragraph 3.3.3]. Let $\kappa^{\prime}$ be its unique $\beta$-extension whose determinant has order a power of $p$. This gives us a bijection $\mathrm{X} / \Gamma \rightarrow \mathcal{D}_{0}(\mathrm{H}, \boldsymbol{\Theta})$, denoted $\boldsymbol{\omega}^{\prime}$. Putting the bijections $\boldsymbol{\omega}, \boldsymbol{\omega}^{\prime}$ and the inertial Jacquet-Langlands correspondence $\boldsymbol{\pi}_{0}$ of (8.1) together, we get a permutation $\Upsilon=\omega^{\prime-1} \circ \boldsymbol{\pi}_{0} \circ \boldsymbol{\omega}$ of $\mathrm{X} / \Gamma$.

Remark 9.1. - This permutation depends a priori on the choice of the ps-character $(\Theta, 0, \beta)$ with endo-class $\boldsymbol{\Theta}$, as well as of that of $\iota, \mathfrak{a}, \iota^{\prime}, \mathfrak{a}^{\prime}$. Under the Compatibility Assumption below, Theorem 9.3 will show that $\Upsilon$ is actually independent of these choices.

We now go back to the simple character $\theta$. Restricting it to the 1-units of $\mathfrak{b}$, it takes the form $\xi_{\theta} \circ \operatorname{Nrd}_{\mathrm{B}}$ for a unique character $\xi_{\theta}$ of the 1-units $1+\mathfrak{p}_{\iota \mathrm{E}}$, where $\operatorname{Nrd}_{\mathrm{B}}$ denotes the reduced norm of B (see $[\mathbf{3 2}, 3.3 .2]$ ). Composing with $\iota$, we get a character $\xi_{\theta} \circ \iota$ of $1+\mathfrak{p}_{\mathrm{E}}$. Similarly, we have a character $\xi_{\theta^{\prime}} \circ \iota^{\prime}$ of the same group.

Compatibility Assumption. We assume that

$$
\xi_{\theta} \circ \iota=\xi_{\theta^{\prime}} \circ \iota^{\prime}
$$

on $1+\mathfrak{p}_{\mathrm{E}}$.

From now on, we assume that the Compatibility Assumption is satisfied. The character (9.2) of the 1-units $1+\mathfrak{p}_{\mathrm{E}}$ will be denoted $\xi_{0}$.

Remark 9.2. - Let $\mathcal{E}(\mathrm{F})$ denote the set of all endo-classes over F, and define $\mathcal{E}(\mathrm{E})$ accordingly. There is a canonical map

$$
\mathcal{E}(\mathrm{E}) \rightarrow \mathcal{E}(\mathrm{F})
$$


given by [8, Corollary 9.3] (see also [15, Chapter 2]), called the restriction map. It is surjective with finite fibers. According to [11, Paragraph 1.2], we can identify E-endo-classes of degree 1 with characters of $1+\mathfrak{p}_{\mathrm{E}}$. The tameness assumption on $\Theta$ implies that the $\mathrm{E} / \mathrm{F}$-lifts of $\Theta$, that is, the endo-classes in $\mathcal{E}(\mathrm{E})$ whose restriction to $\mathcal{E}(\mathrm{F})$ is $\boldsymbol{\Theta}$, all have degree 1 . Besides, $\xi_{0}$ is one of these lifts, and the map $\gamma \mapsto \xi_{0} \circ \gamma$ induces a bijection between $\operatorname{Aut}_{\mathrm{F}}(\mathrm{E})$ and the set of $\mathrm{E} / \mathrm{F}$-lifts of $\Theta$ (see [15, Corollary 2.4])). This gives us a full description of the E/F-lifts of $\Theta$.

Our purpose is to get a formula for $\Upsilon$. In Paragraph 9.4, we will use the results of $[\mathbf{1 3}]$ in order to compute the $\Gamma$-orbit $\Upsilon([\alpha])$ for $\boldsymbol{e}$-regular characters $\alpha \in \mathrm{X}$. We will then use Proposition 8.8 to extend this formula to all characters $\alpha$.

Theorem 9.3. - There is a canonically determined character $\mu$ of $\boldsymbol{k}^{\times}$, depending only on $m$, $d$ and $\Theta$, such that $\mu^{2}=1$ and

$$
\Upsilon([\alpha])=[\alpha \mu]
$$

for all characters $\alpha \in \mathrm{X}$.

More precisely, we will see that the character $\mu$ is the "rectifier" given by Bushnell-Henniart's First Comparison Theorem [13, 6.1] together with [13, Corollary 6.9 and (6.7.4)]. Since the results from [13] we will use are formulated in terms of admissible pairs, we first have to translate them in terms of our $\alpha$-parameters.

Let us write $\mathrm{X}_{\text {reg }}$ for the set of $\boldsymbol{e}$-regular characters in $\mathrm{X}$.

\section{2 .}

We first recall the definition of admissible pairs $[\mathbf{2 4}, \mathbf{1 3}]$, and basic facts about them.

Definition 9.4. - An admissible pair is a pair $(\mathrm{L} / \mathrm{F}, \xi)$ made of a finite, tamely ramified field extension $\mathrm{L} / \mathrm{F}$ and a character $\xi$ of $\mathrm{L}^{\times}$such that:

(1) $\xi$ does not factor through $\mathrm{N}_{\mathrm{L} / \mathrm{K}}$ for any field $\mathrm{K}$ such that $\mathrm{F} \subseteq \mathrm{K} \subsetneq \mathrm{L}$;

(2) if the restriction of $\xi$ to the 1-units $1+\mathfrak{p}_{\mathrm{L}}$ factors through $\mathrm{N}_{\mathrm{L} / \mathrm{K}}$ for some field $\mathrm{K}$ such that $\mathrm{F} \subseteq \mathrm{K} \subseteq \mathrm{L}$, then $\mathrm{L} / \mathrm{K}$ is unramified.

Two admissible pairs $\left(\mathrm{L}_{i} / \mathrm{F}, \xi_{i}\right), i=1,2$, are said to be isomorphic if there is an F-isomorphism $\phi: \mathrm{L}_{2} \rightarrow \mathrm{L}_{1}$ such that $\xi_{2}=\xi_{1} \circ \phi$. The degree of an admissible pair $(\mathrm{L} / \mathrm{F}, \xi)$ is $[\mathrm{L}: \mathrm{F}]$. We also introduce the following definition, which will be convenient to us.

Definition 9.5. - Two admissible pairs $\left(\mathrm{L}_{i} / \mathrm{F}, \xi_{i}\right)$ for $i=1,2$, are said to be inertially equivalent if there are an unramified character $\chi$ of $\mathrm{L}_{2}^{\times}$and an isomorphism $\phi: \mathrm{L}_{2} \rightarrow \mathrm{L}_{1}$ of extensions of $\mathrm{F}$ such that $\chi \xi_{2}=\xi_{1} \circ \phi$. We will write $\left[\mathrm{L}_{1} / \mathrm{F}, \xi_{1}\right]$ for the inertial class of $\left(\mathrm{L}_{1} / \mathrm{F}, \xi_{1}\right)$.

Let $(\mathrm{L} / \mathrm{F}, \xi)$ be an admissible pair. By $[\mathbf{1 3}, 4.1$ Lemma], there is a unique sub-extension $\mathrm{P} / \mathrm{F}$ of $\mathrm{L} / \mathrm{F}$ such that $\xi \mid 1+\mathfrak{p}_{\mathrm{L}}$ factors through the norm $\mathrm{N}_{\mathrm{L} / \mathrm{P}}$ and which is minimal for this property. It is called the parameter field of the admissible pair. Then $\mathrm{L} / \mathrm{P}$ is unramified and, if we write $\xi \mid 1+\mathfrak{p}_{\mathrm{L}}=\xi_{1} \circ \mathrm{N}_{\mathrm{L} / \mathrm{P}}$ for some character $\xi_{1}$ of $1+\mathfrak{p}_{\mathrm{P}}$, then $\left(\mathrm{P} / \mathrm{F}, \xi_{1}\right)$ is an admissible 1-pair in the sense of $[\mathbf{1 3}, 3.3]$, that is, $\xi_{1}$ does not factor through $\mathrm{N}_{\mathrm{L} / \mathrm{K}}$ for any field $\mathrm{K}$ such that $\mathrm{F} \subseteq \mathrm{K} \subsetneq \mathrm{L}$. According to [11, Theorem 1.3], there is a canonical bijective map between isomorphism classes 
of admissible 1-pairs over $\mathrm{F}$ and essentially tame endo-classes over $\mathrm{F}$. Therefore, the admissible 1-pair $\left(\mathrm{P} / \mathrm{F}, \xi_{1}\right)$ determines an essentially tame endo-class, which depends on the inertial class of $(\mathrm{L} / \mathrm{F}, \xi)$ only.

Recall that we have fixed an endo-class $\Theta$ and a character $\xi_{0}$ of $1+\mathfrak{p}_{\mathrm{E}}$ in the Compatibility Assumption of Paragraph 9.1.

Lemma 9.6. - Any admissible pair having endo-class $\Theta$ is isomorphic to an admissible pair $(\mathrm{L} / \mathrm{F}, \xi)$ with associated 1-pair $\left(\mathrm{E} / \mathrm{F}, \xi_{0}\right)$.

Proof. - Suppose that $(\mathrm{L} / \mathrm{F}, \xi)$ has endo-class $\Theta$, and let $\left(\mathrm{P} / \mathrm{F}, \xi_{1}\right)$ be its associated admissible 1-pair. By looking at [11, Paragraph 1.3] in more detail, it follows that $\mathrm{P}$ is $\mathrm{F}$-isomorphic to $\mathrm{E}$. Up to isomorphism, we thus may assume that $\mathrm{P}$ is equal to $\mathrm{E}$. By Remark 9.2, the characters $\xi_{1}, \xi_{0}$ of $1+\mathfrak{p}_{\mathrm{E}}$, which define the same endo-class $\boldsymbol{\Theta}$, are conjugate under the automorphism group $\operatorname{Aut}_{\mathrm{F}}(\mathrm{E})$. Therefore, up to isomorphism, we may assume these characters $\xi_{1}, \xi_{0}$ are equal.

\section{3 .}

Let $(\mathrm{L} / \mathrm{F}, \xi)$ be an admissible pair with endo-class $\Theta$ and degree $t$ dividing $n$. By Lemma 9.6, we may assume that it has associated 1-pair $\left(\mathrm{E} / \mathrm{F}, \xi_{0}\right)$. We may also assume that $\mathrm{L}$ is contained in $\overline{\mathrm{F}}$. By [13, 4.3 Lemma 1], there is a unique character $\xi_{\mathrm{w}}$ of the group of units $\mathcal{O}_{\mathrm{L}}^{\times}$such that:

(1) the characters $\xi_{\mathrm{w}}$ and $\xi$ coincide on the principal unit subgroup $1+\mathfrak{p}_{\mathrm{L}}$;

(2) the order of $\xi_{\mathrm{w}}$ is a power of $p$.

The character $\xi \xi_{\mathrm{w}}^{-1}$ of $\mathcal{O}_{\mathrm{L}}^{\times}$is tamely ramified: it thus induces a character $\xi_{\mathrm{t}}$ of $\boldsymbol{l}^{\times}$, where $\boldsymbol{l}$ is the residue field of $\mathrm{L}$. This character only depends on the inertial class of $(\mathrm{L} / \mathrm{F}, \xi)$.

Since $(\mathrm{L} / \mathrm{F}, \xi)$ is an admissible pair with parameter field $\mathrm{E}$, the residue field $\boldsymbol{l}$ is an extension of $\boldsymbol{e}$ and $\xi_{\mathrm{t}}$ is an $\boldsymbol{e}$-regular character of $\boldsymbol{l}^{\times}$. Since $\mathrm{L} \subseteq \overline{\mathrm{F}}$, the residue field $\boldsymbol{l}$ naturally embeds in $\boldsymbol{k}$. Write $\alpha_{\xi}$ for the character $\xi_{\mathrm{t}} \circ \mathrm{N}_{\boldsymbol{k} / \boldsymbol{l}}$ of $\boldsymbol{k}^{\times}$. Its parametric degree $f$ is equal to [L:E]. We thus have $t=f g$.

We write $\mathcal{P}_{n}(\boldsymbol{\Theta})$ for the set of inertial classes of admissible pairs with endo-class $\boldsymbol{\Theta}$ and degree dividing $n$.

Lemma 9.7. -

(2) The map

induces a bijection between the set of inertial classes of admissible pairs in $\mathcal{P}_{n}(\boldsymbol{\Theta})$ and $\mathrm{X} / \Gamma$.

Remark 9.8. - The map (9.3) depends on the choices we have made in Paragraph 9.1.

Proof. - The character $\alpha_{\xi}$ is $\boldsymbol{e}$-regular if and only if $f=n^{\prime}$. Multiplying by $g$, this is equivalent to $t=n$. This gives us the first part of the lemma.

Given $\alpha \in \mathrm{X}$, there is a uniquely determined field $\boldsymbol{l}$ such that $\boldsymbol{e} \subseteq \boldsymbol{l} \subseteq \boldsymbol{k}$ and $\alpha$ factors through the norm $\mathrm{N}_{\boldsymbol{k} / \boldsymbol{l}}$, and which is minimal for this property. Write $\alpha=\beta \circ \mathrm{N}_{\boldsymbol{k} / \boldsymbol{l}}$ for some character $\beta$ of $\boldsymbol{l}^{\times}$, which is $\boldsymbol{e}$-regular by minimality of $\boldsymbol{l}$. Let L be an unramified extension of $\mathrm{E}$ with residue field $\boldsymbol{l}$. Then $\beta$ inflates to a tamely ramified character of the units subgroup of $\mathrm{L}$, still denoted $\beta$. 
Now write $\xi_{\mathrm{w}}$ for the character of $\mathcal{O}_{\mathrm{L}}^{\times}$of $p$-power order extending the character $\xi_{0} \circ \mathrm{N}_{\mathrm{L} / \mathrm{E}}$ of the 1-units of $\mathrm{L}$, and let $\xi$ be any character of $\mathrm{L}^{\times}$extending $\xi_{\mathrm{w}} \beta$. Since the character $\beta$ is $\boldsymbol{e}$-regular, it follows that the pair $(\mathrm{L} / \mathrm{F}, \xi)$ is admissible. The $\Gamma$-orbit $\left[\alpha_{\xi}\right]$ associated with its inertial class is equal to $[\alpha]$. The map (9.3) is thus surjective.

We now assume that we have two admissible pairs $\left(\mathrm{L}_{i} / \mathrm{F}, \xi_{i}\right)$ for $i=1,2$, with same image $[\alpha]$ in $\mathrm{X} / \Gamma$. For each $i$, we may assume that $\left(\mathrm{L}_{i} / \mathrm{F}, \xi_{i}\right)$ has associated 1 -pair $\left(\mathrm{E} / \mathrm{F}, \xi_{0}\right)$ by Lemma 9.6, and we may further assume that $\mathrm{L}_{i} \subseteq \overline{\mathrm{F}}$. The character $\xi_{i} \mid 1+\mathfrak{p}_{\mathrm{L}_{i}}$ thus factors through $\mathrm{N}_{\mathrm{L}_{i} / \mathrm{E}}$ and $\mathrm{E}$ is minimal for this property. We have an $\boldsymbol{e}$-regular character $\xi_{i, \mathrm{t}}$ of $\boldsymbol{l}_{i}^{\times}$, where $\boldsymbol{l}_{i}$ is the residue field of $\mathrm{L}_{i}$. Since $\left[\alpha_{\xi_{1}}\right],\left[\alpha_{\xi_{2}}\right]$ are equal, they have the same cardinality $f$. The fields $\boldsymbol{l}_{1}$, $\boldsymbol{l}_{2}$ thus have the same degree over $\boldsymbol{e}$, and $\mathrm{L}_{1}, \mathrm{~L}_{2}$ have the same degree $f$ over $\mathrm{E}$. We thus have $\mathrm{L}_{1}=\mathrm{L}_{2}$, denoted L. We now have two characters $\xi_{1, \mathrm{t}}$ and $\xi_{2, \mathrm{t}}$ of $\boldsymbol{l}^{\times}$, which are conjugate under $\mathrm{Gal}(\boldsymbol{l} / \boldsymbol{e})$. Changing again $\left(\mathrm{L}_{2} / \mathrm{F}, \xi_{2}\right)$ in its isomorphism class, we may assume that they are equal. Thus the admissible pairs $\left(\mathrm{L}_{i} / \mathrm{F}, \xi_{i}\right)$, for $i=1,2$, are inertially equivalent.

\section{4 .}

The Parametrization Theorem [13, 6.1] gives us a canonical bijection

$$
(\mathrm{L} / \mathrm{F}, \xi) \mapsto \Pi(\mathrm{G}, \xi)
$$

between isomorphism classes of admissible pairs of degree $n$ and isomorphism classes of essentially tame irreducible cuspidal representations of $\mathrm{G}$ (that is, cuspidal representations with essentially tame endo-class) of parametric degree $n$.

Lemma 9.9. - (1) Given an admissible pair $(\mathrm{L} / \mathrm{F}, \xi)$ of degree $n$ and with associated 1-pair $\left(\mathrm{E} / \mathrm{F}, \xi_{0}\right)$, the irreducible cuspidal representation $\Pi(\mathrm{G}, \xi)$ belongs to the inertial class $\Omega\left(\kappa, \alpha_{\xi}\right)$.

(2) The bijection (9.4) induces a bijection between inertial classes of admissible pairs of degree $n$ and inertial classes of essentially tame cuspidal representations of $\mathrm{G}$ of parametric degree $n$.

Proof. - By examining the construction of [13, 4.2 and 4.3], we see that an essentially tame irreducible cuspidal representation of endo-class $\Theta$ will correspond through (9.4) to an admissible pair $(\mathrm{L} / \mathrm{F}, \xi)$ with associated 1-pair $\left(\mathrm{E} / \mathrm{F}, \xi_{0}\right)$ if and only if it contains the maximal simple type $\kappa \otimes \sigma$, where $\sigma$ is the irreducible cuspidal representation of $\mathcal{G}$ whose Green parameter in $\mathrm{X} / \Gamma$ is $\left[\alpha_{\xi}\right]$. Comparing with the construction of Paragraph 3.3, the simple type $\kappa \otimes \sigma$ is $\boldsymbol{\lambda}\left(\alpha_{\xi}\right)$. This gives us the first part of the lemma.

An inertial class of essentially tame cuspidal representations of $\mathrm{G}$ with endo-class $\Theta$ has the form $\Omega(\kappa, \alpha)$ for some $\alpha \in \mathrm{X}_{\mathrm{reg}}$. The second part of the lemma thus follows from Lemma 9.7.

We now prove Theorem 9.3 for $\boldsymbol{e}$-regular characters of X.

Proposition 9.10. - (1) There is a canonically determined character $\mu \in \mathrm{X}$, depending on $m, d$ and $\Theta$ only, such that $\mu^{2}=1$ and

$$
\Upsilon([\alpha])=[\alpha \mu]
$$

for all characters $\alpha \in \mathrm{X}_{\mathrm{reg}}$. 
(2) The character $\mu$ is non-trivial if and only if $p \neq 2$ and the integer

$$
y(\boldsymbol{\Theta}, m, d)=m(d-1)+m^{\prime}\left(d^{\prime}-1\right)+u(v-1)
$$

is odd, where the integers $u, v \geqslant 1$ are defined by $u v=n / w, v=d /(d, w)$ with $w=n / e(\mathrm{E} / \mathrm{F})$.

Proof. - Let $\alpha \in \mathrm{X}_{\text {reg }}$ and let $(\mathrm{L} / \mathrm{F}, \xi)$ be an admissible pair of degree $n$ and endo-class $\boldsymbol{\Theta}$ whose inertial class is associated with $[\alpha]$. By $[\mathbf{1 3}$, Theorem $\mathrm{A}]$, there is a tamely ramified character $\nu$ of $\mathrm{L}^{\times}$such that $(\mathrm{L} / \mathrm{F}, \xi \nu)$ is admissible, $\nu^{2}$ is trivial and the Jacquet-Langlands transfer of $\Pi(\mathrm{G}, \xi)$ is $\Pi(\mathrm{H}, \xi \nu)$.

Now suppose that $(\mathrm{L} / \mathrm{F}, \xi)$ has associated 1-pair $\left(\mathrm{E} / \mathrm{F}, \xi_{0}\right)$ and $\mathrm{L}$ is contained in $\overline{\mathrm{F}}$. Since $\mathrm{L} / \mathrm{F}$ has degree $n$ and $\mathrm{L}$ is unramified over $\mathrm{E}$, the residue field of $\mathrm{L}$ canonically identifies with $\boldsymbol{k}$. We write $\mu$ for the character of $\boldsymbol{k}^{\times}$induced by the restriction of $\nu$ to the units subgroup of L. This character is entirely described by [13, Corollary 6.9], which gives us Part 2 of the proposition.

Taking inertial classes and using Lemma 9.9, the Jacquet-Langlands correspondence matches together the inertial class $\Omega(\kappa, \alpha)$ of $\Pi(\mathrm{G}, \xi)$ with that of $\Pi(\mathrm{H}, \xi \nu)$, and the latter can be written $\Omega\left(\kappa^{\prime}, \alpha^{\prime}\right)$ for $\left[\alpha^{\prime}\right]=\left[\alpha_{\xi \nu}\right]=[\alpha \mu]$. The result follows.

\section{5 .}

We now prove Theorem 9.3. Following Remark 8.7, let us fix an odd integer $a \geqslant 7$. We will see below why it is convenient to choose $a$ odd. We use the notation introduced in Paragraph 8.3. In particular, we have $\beta$-extensions $\kappa^{*}, \kappa^{*}$ and a permutation $\Upsilon^{*}$ of $\mathrm{X}^{*} / \Gamma^{*}$. We must pay attention to the fact that the determinants of $\kappa^{*}, \kappa^{* *}$ have orders which may not be powers of $p$, thus Proposition 9.10 may not apply to $\Upsilon^{*}$ directly.

Let us write $\kappa_{p}^{*}$ for the $\beta$-extension on $\mathrm{J}\left(\mathfrak{a}^{*}, \iota \beta\right)$ whose determinant has order a power of $p$. By Remark 3.11 there is a character $\zeta$ of $\mathrm{J}\left(\mathfrak{a}^{*}, \iota \beta\right)$ trivial on $\mathrm{J}^{1}\left(\mathfrak{a}^{*}, \iota \beta\right)$ such that $\kappa_{p}^{*}=\kappa^{*} \zeta$. This induces a character of $\mathrm{GL}_{m^{\prime}}(\boldsymbol{d})$ of the form $\chi \circ \phi_{\iota} \circ \mathrm{N}_{\boldsymbol{d} / \boldsymbol{e}_{\iota}} \circ$ det for some character $\chi$ of $\boldsymbol{e}^{\times}$.

Similarly, we have a $\beta$-extension $\kappa_{p}^{\prime *}$ whose determinant has order a power of $p$, and characters $\zeta^{\prime}, \chi^{\prime}$ such that $\kappa_{p}^{\prime *}=\kappa^{\prime *} \zeta^{\prime}$ and $\zeta^{\prime}$ induces the character $\chi^{\prime} \circ \phi_{\iota^{\prime}} \circ \operatorname{det}$ of $\mathrm{GL}_{n^{\prime}}\left(\boldsymbol{e}_{\iota^{\prime}}\right)$. We write $\Psi_{p}$ for the permutation of $\mathrm{X}^{*} / \Gamma^{*}$ corresponding to the maximal $\beta$-extensions $\kappa_{p}^{*}$ and $\kappa_{p}^{\prime *}$. We write $\delta$ for the character $\left(\chi^{\prime} \chi^{-1}\right) \circ \mathrm{N}_{\boldsymbol{k}^{*} / \boldsymbol{e}} \in \mathrm{X}^{*}$.

Lemma 9.11. - The character $\delta$ is trivial.

Proof. - Let $\beta \in \mathrm{X}^{*}$ be an $\boldsymbol{e}$-regular character. Applying Proposition 9.10 to $\Psi_{p}$ gives us the equality $\Psi_{p}([\beta])=[\beta \lambda]$ where $\lambda \in \mathrm{X}^{*}$ is the rectifying character corresponding to $a m, d$ and $\boldsymbol{\Theta}$. Since $a$ has been chosen to be odd, we have

$$
y(\boldsymbol{\Theta}, a m, d) \equiv y(\boldsymbol{\Theta}, m, d) \quad \bmod 2 .
$$

It follows that $\lambda$ is trivial if and only if $\mu$ is, that is $\lambda=\mu^{*}$. We thus get $\Psi_{p}([\beta])=\left[\beta \mu^{*}\right]$.

Now let $\varepsilon$ be the character $\chi \circ \mathrm{N}_{\boldsymbol{k}^{*} / \boldsymbol{e}}$ and define $\varepsilon^{\prime}$ similarly. Comparing $\Psi_{p}$ and $\Upsilon^{*}$ thanks to Remark 3.11, we get $\Upsilon^{*}([\beta \varepsilon])=\left[\beta \mu^{*} \varepsilon^{\prime}\right]$ for all $\boldsymbol{e}$-regular character $\beta \in \mathbf{X}^{*}$. Since $\beta \varepsilon^{-1}$ is $\boldsymbol{e}$-regular if and only if $\beta$ is, this gives us

$$
\Upsilon^{*}([\beta])=\left[\beta \delta \mu^{*}\right]
$$


for all $\boldsymbol{e}$-regular $\beta \in \mathrm{X}^{*}$.

Now let $\alpha \in \mathrm{X}_{\text {reg. }}$. By Lemma 8.5 there are a prime number $\ell \neq p$ not dividing the order of $\boldsymbol{k}^{\times}$and an $\boldsymbol{e}$-regular character $\beta \in \mathrm{X}^{*}$ such that $\beta \equiv \alpha^{*} \bmod \ell$. By (9.5) and Proposition 8.8 we get $\Upsilon([\alpha])=[\alpha \nu]$ for some $\nu \in \mathrm{X}$ such that $\nu^{*}$ is the $\ell$-regular part of $\delta \mu^{*}$. Since $\alpha$ is $\boldsymbol{e}$-regular, Proposition 9.10 applied to $\Upsilon$ gives us $\Upsilon([\alpha])=[\alpha \mu]$. Putting these equalities together, we get

$$
\left[\alpha^{*} \mu^{*}\right] \equiv\left[\alpha^{*} \mu^{*} \delta\right] \bmod \ell
$$

The character $\delta$ can thus be written $\xi\left(\alpha^{*} \mu^{*}\right)^{\mathrm{Q}^{i}-1}$ for some integer $i \in\left\{0, \ldots, n^{\prime}-1\right\}$ and some $\xi \in \mathrm{X}^{*}$ whose order is a power of $\ell$. (Recall that Q is the cardinality of $\boldsymbol{e}$.) Since $\mu$ has order at most 2 , we get $\delta=\xi\left(\alpha^{*}\right)^{\mathrm{Q}^{i}-1}$. Since the orders of $\delta$ and $\mu^{*}$ both divide $\mathrm{Q}-1$, we have

$$
\alpha^{*\left(\mathrm{Q}^{i}-1\right)(\mathrm{Q}-1)}=\xi^{1-\mathrm{Q}} .
$$

Since both $\mathrm{Q}-1$ and the order of $\alpha$ are prime to $\ell$, we get $\xi=1$. Thus the order of $\alpha$, that we may assume to be $\mathrm{Q}^{n^{\prime}}-1$ by choosing for $\alpha$ a generator of $\mathrm{X}$, divides $\left(\mathrm{Q}^{i}-1\right)(\mathrm{Q}-1)$. This implies $i=0$, thus $\delta$ is trivial as expected.

Now let $\alpha \in \mathrm{X}$ be arbitrary. By Lemma 8.5 there are a prime number $\ell \neq p$ not dividing the order of $\boldsymbol{e}[\alpha]^{\times}$and an $\boldsymbol{e}$-regular character $\beta \in \mathrm{X}^{*}$ such that $\beta$ is congruent to $\alpha^{*} \bmod \ell$. Since $\delta=\mu^{*}$, the identity (9.5) gives us $\Upsilon^{*}([\beta])=\left[\beta \mu^{*}\right]$. By Proposition 8.8, we have $\Upsilon([\alpha])=[\alpha \nu]$ for some character $\nu \in \mathrm{X}$ such that $\nu^{*}$ is the $\ell$-regular part of $\mu^{*}$. Thus $\nu^{*}=\mu^{*}$, which implies $\nu=\mu$. This completes the proof of Theorem 9.3.

Corollary 9.12. - The permutation $\Upsilon$ does not depend on the choice of the F-embeddings $\iota, \iota^{\prime}$ and the orders $\mathfrak{a}, \mathfrak{a}^{\prime}$, nor on the choice of the ps-character $(\Theta, 0, \beta)$ of endo-class $\boldsymbol{\Theta}$.

\section{6 .}

We now translate Theorem 9.3 in terms of admissible pairs. Let $(\mathrm{L} / \mathrm{F}, \xi)$ be an admissible pair of degree dividing $n$ and endo-class $\boldsymbol{\Theta}$. The orbit $\left[\alpha_{\xi}\right] \in \mathrm{X} / \Gamma$ given by (9.3) corresponds through (9.1) to an inertial class $\Omega\left(\kappa, \alpha_{\xi}\right)$ of discrete series representations. Write $\Pi_{0}(\mathrm{G}, \xi)$ for this inertial class. The map

$$
[\mathrm{L} / \mathrm{F}, \xi] \mapsto \Pi_{0}(\mathrm{G}, \xi)
$$

is a bijection between $\mathcal{P}_{n}(\boldsymbol{\Theta})$ and $\mathcal{D}_{0}(\mathrm{G}, \boldsymbol{\Theta})$. This map depends a priori on various choices.

Theorem 9.13. - Let $(\mathrm{L} / \mathrm{F}, \xi)$ be an admissible pair with degree dividing $n$. There is a canonically determined tamely ramified character $\mu$ of the units subgroup of $\mathrm{L}$ such that $\mu^{2}=1$ and

$$
\boldsymbol{\pi}_{0}\left(\Pi_{0}(\mathrm{G}, \xi)\right)=\Pi_{0}(\mathrm{H}, \xi \mu) .
$$

It depends only on $m, d$ and the restriction of $\xi$ to the principal units $1+\mathfrak{p}_{\mathrm{L}}$.

Note that by $\Pi_{0}(\mathrm{H}, \xi \mu)$ we mean the inertial class corresponding to the pair $[\mathrm{L} / \mathrm{F}, \xi \hat{\mu}]$ for any choice of extension $\hat{\mu}$ of $\mu$ to $\mathrm{L}^{\times}$; this is independent of the choice of $\hat{\mu}$. 
Remark 9.14. - Let $t$ be the degree of $\mathrm{L} / \mathrm{F}$ and write $s$ for the integer $s\left(\alpha_{\xi}\right)$ defined by (8.3). The parametric degree $f=[\mathrm{L}: \mathrm{E}]$ of $\alpha_{\xi}$ divides $m^{\prime} d^{\prime}$. Hence $u=f /\left(f, d^{\prime}\right)$ divides $m^{\prime} s$, thus $m^{\prime}$. Let us define an integer $r \geqslant 1$ by $m^{\prime}=u r$, or equivalently by $n=r s t$. Any discrete series representation in $\Pi_{0}(\mathrm{G}, \xi)$ has the form $\mathrm{L}(\rho, r)$ for some cuspidal representation $\rho$ of $\mathrm{GL}_{m / r}(\mathrm{D})$ with parametric degree $t$.

Remark 9.15. - An admissible pair $(\mathrm{L} / \mathrm{F}, \xi)$ of degree $t$ dividing $n$ canonically defines, via the canonical map (9.4), an isomorphism class of essentially tame cuspidal representation $\rho_{\xi}$ of the group $\mathrm{GL}_{t}(\mathrm{~F})$. Passing to inertial classes, the map

$$
(\mathrm{L} / \mathrm{F}, \xi) \mapsto \mathrm{L}\left(\rho_{\xi}, \frac{n}{t}\right)
$$

induces the map (9.6) when $\mathrm{G}=\mathrm{H}$, which is thus canonical in that case. It follows from Theorem 9.13 that (9.6) is a canonical bijection between $\mathcal{P}_{n}(\boldsymbol{\Theta})$ and $\mathcal{D}_{0}(\mathrm{G}, \boldsymbol{\Theta})$, for any inner form $\mathrm{G}$.

\section{References}

[1] A. I. Badulescu, Correspondance de Jacquet-Langlands en caractéristique non nulle, Ann. Scient. Éc. Norm. Sup. (4) 35 (2002), 695-747.

[2] A. I. Badulescu, Jacquet-Langlands et unitarisabilité, J. Inst. Math. Jussieu 6 (2007), n³, 349-379.

[3] P. Broussous, Extension du formalisme de Bushnell et Kutzko au cas d'une algèbre à division, Proc. London Math. Soc. (3) 77 (1998), n², 292-326.

[4] _ Transfert du pseudo-coefficient de Kottwitz et formules de caractère pour la série discrète de $\mathrm{GL}(N)$ sur un corps local, Canadian Journal of Mathematics 66 (2014), n², 241-283.

[5] P. Broussous and P. Schneider, Type theory and coefficient systems on the building, Bull. Soc. Math. France 145 (2017), $\mathrm{n}^{\circ} 1,97-159$.

[6] P. Broussous, V. Sécherre and S. Stevens, Smooth representations of $\mathrm{GL}_{m}(\mathrm{D})$, V: endo-classes, Documenta Math. 17 (2012), 23-77.

[7] C. J. Bushnell and G. Henniart, Correspondance de Jacquet-Langlands explicite II : le cas de degré égal à la caractéristique résiduelle, Manuscripta Math. 102 (2000), 211-225.

[8] _ Local tame lifting for GL(N), I: simple characters, Publ. Math. Inst. Hautes Études Sci. 83 (1996), 105-233.

[9] Local tame lifting for GL(N), IV: simple characters and base change, J. London Math. Soc. 87 (2003), 337-362.

[10] _ Local tame lifting for GL(N), III: explicit base change and Jacquet-Langlands correspondence, J. reine angew. Math. 580 (2005), 39-100.

[11] _ The essentially tame local Langlands correspondence, I, Journal of the Amer. Math. Soc. 18 (2005), $\mathrm{n}^{\circ} 3,685-710$.

[12] _ The essentially tame local Langlands correspondence, III: the general case, Proc. Lond. Math. Soc. (3) 101 (2010), n ${ }^{\circ}$, 497-553.

[13] _ The essentially tame Jacquet-Langlands correspondence for inner forms of GL(n), Pure Appl. Math. Q. 7 (2011), n³, 469-538.

[14] _ Explicit functorial correspondences for level 0 representations of p-adic linear groups, J. Number Theory 131 (2011), 309-331.

[15] _ To an effective local Langlands correspondence, Mem. Amer. Math. Soc. 231 (2014).

[16] C. J. Bushnell and P. C. Kutzko, The admissible dual of GL(N) via compact open subgroups, Princeton University Press, Princeton, NJ, 1993. 
[17] J.-F. Dat, Un cas simple de correspondance de Jacquet-Langlands modulo , Proc. London Math. Soc. 104 (2012), 690-727.

[18] P. Deligne, D. Kazhdan and M.-F. Vignéras, Représentations des algèbres centrales simples p-adiques, Representations of reductive groups over a local field, Hermann, Paris, 1984.

[19] R. Dipper, On the decomposition numbers of the finite general linear groups. II, Trans. Amer. Math. Soc. 292 (1985), $\mathrm{n}^{\circ} 1,123-133$.

[20] A. Dotto, The inertial Jacquet-Langlands correspondence, preprint (2017).

[21] J. A. Green, The characters of the finite general linear groups, J. Algebra $184(1996), \mathrm{n}^{\circ} 3$, 839-851. $^{2}$

$[22]$ D. Helm, The Bernstein center of the category of smooth $\mathrm{W}(k)\left[\mathrm{GL}_{n}(F)\right]$-modules, Forum Math. Sigma 4 (2016).

[23] G. Henniart, Correspondance de Jacquet-Langlands explicite I : le cas modéré de degré premier, in Séminaire de théorie des nombres de Paris 1990-91, Progress in Mathematics 38, Birkhäuser, 1993.

[24] R. E. Howe, Tamely ramified supercuspidal representations of $\mathrm{GL}_{n}$, Pacific J. Math. 73 (1977), 437-460.

[25] N. Imai and T. Tsushima, Local Jacquet-Langlands correspondences for simple supercuspidal representations, Kyoto J. Math. 58 (2018), n³, 623-638.

[26] H. Jacquet and R. P. Langlands, Automorphic forms on GL(2), Lecture Notes in Mathematics 114, Springer, 1970.

[27] A. Mínguez and V. Sécherre, Représentations lisses modulo $\ell$ de $\mathrm{GL}_{m}$ (D), Duke Math. J. 163 (2014), 795-887.

[28] _ Types modulo $\ell$ pour les formes intérieures de $\mathrm{GL}_{n}$ sur un corps local non archimédien, avec un appendice par V. Sécherre et S. Stevens, Proc. London Math. Soc. 109 (2014), n 4, p. 823-891.

[29]_, Représentations modulaires de $\mathrm{GL}_{n}(q)$ en caractéristique non naturelle, Trends in number theory, Contemporary Math. 649 (2015), 163-183.

[30] _ Correspondance de Jacquet-Langlands locale et congruences modulo $\ell$, Invent. Math. 208 (2017), n², 553-631.

[31] J. Rogawski, Representations of $\mathrm{GL}(n)$ and division algebras over a p-adic field, Duke Math. J. 50 (1983), 161-196.

[32] V. Sécherre, Représentations lisses de $\mathrm{GL}_{m}(\mathrm{D})$, I : caractères simples, Bull. Soc. math. France 132 (2004), n $3,327-396$.

[33] _ Représentations lisses de $\mathrm{GL}_{m}(\mathrm{D}), \mathrm{II}$ : $\beta$-extensions, Compositio Math. 141 (2005), 15311550.

[34] _ Représentations lisses de $\mathrm{GL}_{m}(\mathrm{D})$, III : types simples, Ann. Scient. Éc. Norm. Sup. 38 (2005), 951-977.

[35] V. Sécherre and S. Stevens, Représentations lisses de $\mathrm{GL}_{m}(\mathrm{D}), \mathrm{IV}$ : représentations supercuspidales, J. Inst. Math. Jussieu 7 (2008), n 3 , p. 527-574.

[36] _ Smooth representations of $\mathrm{GL}_{m}(\mathrm{D})$, VI: semisimple types, Int. Math. Res. Not. 13 (2012), 2994-3039.

[37] Block decomposition of the category of $\ell$-modular smooth representations of $\mathrm{GL}_{n}(\mathrm{~F})$ and its inner forms, Ann. Scient. Éc. Norm. Sup. 49 (2016), n³, 669-709.

[38] A. J. Silberger and E.-W. Zink, The characters of the generalized Steinberg representations of finite general linear groups on the regular elliptic set, Trans. Amer. Math. Soc. 352 (2000), n 7, 3339-3356.

[39] _ Weak explicit matching for level zero discrete series of unit groups of $\mathfrak{p}$-adic simple algebras, Canad. J. Math. 55 (2003), $\mathrm{n}^{\circ} 2,353-378$.

[40] _ An explicit matching theorem for level zero discrete series of unit groups of $\mathfrak{p}$-adic simple algebras, J. Reine Angew. Math. 585 (2005), 173-235.

[41] M. Tadić, Induced representations of $\mathrm{GL}(n, A)$ for $p$-adic division algebras $A$, J. Reine Angew. Math. 405 (1990), 48-77. 
[42] M.-F. Vignéras, Représentations $l$-modulaires d'un groupe réductif $p$-adique avec $l \neq p$, Progress in Mathematics, vol. 137, Birkhäuser Boston Inc., Boston, MA, 1996.

[43] On highest Whittaker models and integral structures, Contributions to Automorphic forms, Geometry and Number theory: Shalikafest 2002, John Hopkins Univ. Press, 2004, 773-801.

[44] A. Zelevinski, Induced representations of reductive $\mathfrak{p}$-adic groups. II. On irreducible representations of GL(n), Ann. Sci. École Norm. Sup. (4) 13 (1980), n², 165-210.

[45] K. Zsigmondy, Zur Theorie der Potenzreste, Monatsh. Math. 3 (1892) 265-284.

VinCent SÉcherRe, Laboratoire de Mathématiques de Versailles, UVSQ, CNRS, Université Paris-Saclay, 78035 Versailles, France • E-mail : vincent.secherre@math.uvsq.fr

Shaun Stevens, School of Mathematics, University of East Anglia, Norwich NR4 7TJ, United Kingdom E-mail : Shaun.Stevens@uea.ac.uk 